\title{
An anisotropic phase-field model for solid-state dewetting and its sharp-interface limit
}

\author{
Marion Dziwnik ${ }^{\dagger}$ \\ Andreas Münch ${ }^{\ddagger}$ \\ February 16, 2017
}

Barbara Wagner ${ }^{\dagger \S}$

\begin{abstract}
We propose a two-dimensional phase field model for solid state dewetting where the surface energy is weakly anisotropic. The evolution is described by the Cahn-Hilliard equation with a bi-quadratic degenerate mobility together with a bulk free energy based on a doublewell potential and a free boundary condition at the film-substrate contact line. We derive the corresponding sharp interface limit via matched asymptotic analysis involving multiple inner layers. We show that in contrast to the frequently used quadratic degenerate mobility, the resulting sharp interface model for the bi-quatratic mobility is consistent with the pure surface diffusion model. In addition, we show that natural boundary conditions at the substrate obtained from the first variation of the total free energy including contributions at the substrate imply a contact angle condition in the sharp-interface limit which recovers the Young-Herring equation in the anisotropic and Young's equation in the isotropic case, as well as a balance of fluxes at the contact line (or contact point).
\end{abstract}

Key words. phase-field model, matched asymptotic expansions, exponential asymptotics, sharp interface model, free boundary problems, dewetting solid films

AMS subject classification 76Dxx, 76Txx, 35B40, 35C20, 49Jxx

\section{Introduction}

Dewetting of solid films is one of the important processes used for nanostructuring and functionalizing surfaces for a variety of technological applications, such as for example in thin-film solar cells and other optoelectronic devices. Examples can be found in $[52,14,13]$ and for a recent review we refer to Thompson [56]. Typically, the dewetting scenario begins with the formation of a three-phase contact line between the thin solid film, the solid substrate and the surrounding vapor phase. The subsequent retraction of the film leads to the formation of a rim that eventually destabilizes into nano- or micro- islands [57].

While the dynamical evolution has many similarities with the dewetting of liquid thin films, which has been investigated in numerous theoretical and experimental studies $[50,51,28,2]$ and recently reviewed in [8], solid dewetting has not received as much attention. The physical mechanisms for the mass transport underlying the dewetting of solid films is also quite different and it is based on capillarity driven surface diffusion $[61,26,57]$. In addition, further properties

\footnotetext{
$\S$ Weierstrass Institute, Mohrenstraße 39, 10117 Berlin, Germany

$\ddagger$ Mathematical Institute, University of Oxford, Andrew Wiles Building, Woodstock Road, Oxford, OX2 6GG

${ }^{\dagger}$ Technische Universität Berlin, Institute of Mathematics, Straße des 17. Juni 136, 10623 Berlin, Germany
} 
such as anisotropy of its surface energy can dominate the dynamics [65, 15, 58]. This can have important implication on the stability of the moving three-phase contact-line, where vapor, solid film and solid substrate meet. For the equilibrium state the equations governing the shape of a nano- or micro crystal in contact with a substrate has been systematically derived as well as experimentally validated in $[59,31]$.

Since the dynamical dewetting process usually involves a succession of topological transitions of the thin dewetting film, the phase field framework provides an adequate modelling approach for a continuum description that allows the creation and vanishing of interfaces to occur naturally as part of the solution. This is in contrast to interface tracking methods used for sharp-interface models.

Establishing the correct correspondence between the phase-field and sharp-interface models has therefore been investigated intensively during the last decades, see for example the review by [42]. One of the first systematic derivations of sharp-interface models using matched asymptotic expansions has been carried out by Pego [47]. His analysis concerned the Cahn-Hilliard equation for a conserved order parameter $u(\mathbf{x}, t)$

$$
\partial_{t} u=\nabla \cdot \mathbf{j}, \quad \mathbf{j}=m(u) \nabla \mu, \quad \mu=F^{\prime}(u)-\epsilon^{2} \Delta u,
$$

for $\mathbf{x}$ in a domain $\Omega$ in $\mathbb{R}^{N}$, for time $t>0$, and with the homegeneous free energy $F(u)=$ $\frac{1}{2}\left(1-u^{2}\right)^{2}$ and constant mobility $m(u)=1$. The boundary conditions in [47] may be taken to be of homogeneous Neumann type, i.e. $\mathbf{n} \cdot \nabla \mu=\mathbf{n} \cdot \nabla u=0$, where $\mathbf{n}$ is the outward unit normal to $\partial \Omega$, or of Dirichlet type, i.e. $\mu=\mu_{b}, u=u_{b}$, on $\partial \Omega$, where $\partial \Omega$ should be of suitable regularity. For time $t=0$, arbitrary, smooth initial values $u(\mathbf{x}, 0)$ are permitted which describe a smooth interface $\Gamma_{0}$, independent of $\epsilon$, and subject only to the restriction that at any point, whose distance from $\Gamma_{0}$ is greater than $\epsilon$, the corresponding values of $u(\mathbf{x}, 0)$ are sufficiently stable. At distances greater than $O(\epsilon)$ the derivatives will be presumed to be bounded independent of $\epsilon$ as $\epsilon$ tends to zero. For this model Pego recovered on the time scale $t=O\left(\epsilon^{-1}\right)$ the Mullins-Sekerka problem [41], for which the interface motion is driven by pure bulk diffusion. Rigorous treatments using ideas from matched asymptotic expansions were given by Alikakos et al. [1] and for radially symmetric stationary solutions by Niethammer [43].

The particular choice for $F(u)=\frac{1}{2}\left(1-u^{2}\right)^{2}$ and $m(u)=1$ is actually an approximation of the Cahn-Hilliard equation derived in [45] with the concentration dependent degenerate mobility $m(u)=1-u^{2}$ and the logarithmic free energy

$$
F(u)=\frac{T}{2}((1+u) \ln (1+u)+(1-u) \ln (1-u))+\frac{1}{2}\left(1-u^{2}\right)^{2}
$$

in the limit $T \rightarrow 1$, where $T$ is the temperature. For the deep quench limit, $T=0$, and for $T=O\left(\epsilon^{\alpha}\right)$ with $\alpha>0$ Cahn et al. [7] considered the sharp interface limit $\epsilon \rightarrow 0$ and obtained Mullins' model for surface diffusion [40].

Phase-field models combining other approximations of the bulk free energy and the mobility have frequently been investigated as candidates for sharp-interface models driven by surface diffusion in the limit $\epsilon \rightarrow 0$, for example the biquadratic free energy $F(u)=\frac{1}{2}\left(1-u^{2}\right)^{2}$ combined with the degenerate mobility $m(u)=1-u^{2}$ or the biquadratic degenerate mobility as $m(u)=$ $\left(1-u^{2}\right)^{2}$, see for example the studies [25, 53]. However, as has been pointed out by Guggenberger et al. [20] and more recently by Dai et al. [9, 10] the standard matched asymptotic derivations that recover Mullins' model with pure surface diffusion lead to inconsistencies that appear in the asymptotic derivations except when the interface is flat. Indeed, in Lee et al. [34, 33] it was shown that for the combination $F(u)=\frac{1}{2}\left(1-u^{2}\right)^{2}$ and $m(u)=1-u^{2}$ a careful asymptotic analysis involving exponential asymptotics is necessary to resolve this problem and in fact yields to leading order in $\epsilon$ a sharp interface model where both surface and bulk diffusion are present. 
The fact that nonlinear bulk diffusion and surface diffusion contribute to the interfacial mass flux at the same order implies that the phase field model describes a different driving mechanism for the interface evolution than intended (i.e. than in Mullins' model) and this fact has implications for phase-field models intended to describe a particular physical process.

Such is the case for the problem of solid state dewetting. For the isotropic case, a phase-field model has been proposed by Jiang et al. [25] with a phase-field variable $u=u(\mathbf{x}, t)$ that is defined on the domain $\Omega$ and where $u(\mathbf{x}, t)>0, u(\mathbf{x}, t)<0$ and $u(\mathbf{x}, t)=0$ characterise the solid (or film) phase, the vapor phase, and the location of the interface, respectively. For this phase-field variable the total free energy

$$
W^{\epsilon}=\int_{\Omega} f_{F V} d \Omega+\int_{\Gamma_{w}} f_{w} d \Gamma
$$

combines a bulk contribution from the Ginzburg-Landau free energy density

$$
f_{F V}=\lambda_{m}\left(F(u)+\frac{\epsilon^{2}}{2}|\nabla u|\right)
$$

with a surface energy density contribution from the contact line at the substrate $\Gamma_{w} \subset \partial \Omega$,

$$
f_{w}=\frac{\sigma_{V S}+\sigma_{F S}}{2}-\frac{u\left(3-u^{2}\right)}{4}\left(\sigma_{V S}-\sigma_{F S}\right) .
$$

The width of the diffuse interface layer is proportional to $\epsilon, \lambda_{m}$ denotes the mixing energy density and $\sigma_{V S}$ and $\sigma_{F S}$ the vapor-substrate and film-substrate interface energy densities, respectively. A derivation via the first variational derivative of the total free energy functional with respect to $u$, following for example [45], yields the corresponding chemical potential $\mu=\left(1 / \lambda_{m}\right) \delta W^{\epsilon} / \delta u$, so that by making use of the fact that $u$ is a conserved order parameter, the Cahn-Hilliard equation (1) is obtained together with a no-flux boundary condition on $\partial \Omega$. In Jiang et al. [25] for example, the choice $m(u)=1-u^{2}$ and $F(u)=\frac{1}{2}\left(1-u^{2}\right)^{2}$ was suggested to correspond to the sharp-interface model for pure surface diffusion that reflects the underlying physical mechanism of the solid dewetting process. However, since the asymptotic limit does not yield this result, we suggest the mobility of form $m(u)=\left(1-u^{2}\right)^{2}$ and show that this indeed yields the desired sharp-interface model, that is with pure surface diffusion to leading order.

As a model for the wall energy we also include (4) as suggested in [24, 64]. As discussed in [21], it is convenient to choose $f_{w}$ such that away from the contact line, $f_{w}$ gives the vapor/substrate interfacial energy in the vapor phase, i.e. $f_{w}=\gamma_{V S}$, when $u=-1$, and the film/surface interfacial energy in the film phase, i.e. $f_{w}=\gamma_{F S}$, when $u=1$. Moreover $f_{w}$ has to satisfy $f_{w}^{\prime}( \pm 1)=0$, which provides that the energy minimizing solution of the free energy part, i.e. $\int_{\Omega} f_{F V}$, is undisturbed by $f_{w}$. The cubic form of $f_{w}$ is in particular mathematically convenient since it naturally provides the above mentioned properties. Other physically motivated expressions for the wall energy can be found in [48].

In addition, our phase-field model also includes an anisotropic surface energy $\gamma$, which in two space dimensions can be represented by $\gamma(\theta)$, where $\theta$ is the interface orientation angle. We note that anisotropic surface energy may lead to an ill-posed problem when there are missing orientations in the corresponding Wulff shape. If $\gamma^{2}|\nabla u|^{2}$ is not convex then the term $\nabla u$ may be backwards diffusive for some initial data $[60,16]$. In particular, in the two-dimensional case which we consider here, $\nabla u$ is backwards diffusive precisely when $\gamma(\theta)+\gamma^{\prime \prime}(\theta)<0$. This case is referred to as strongly anisotropic and has been investigated by Cahn and Taylor [6], Eggleston et al. [16] suggesting various convexification schemes and has been numerically treated for example by Wise et al. [60] to solve the regularized, anisotropic Cahn-Hilliard equation. 
For weak anisotropy different Cahn-Hilliard models were studied by McFadden et al. [37], considering the solidification process of a pure material, and Rätz et al. [49] for the surface evolution of elastically stressed films, where in both cases the method of matched asymptotic expansions is used to recover the appropriate anisotropic form of the Gibbs-Thomson equation in the sharp interface limit. i.e.

$$
\mu \propto\left(\gamma+\gamma^{\prime \prime}\right) \kappa
$$

where $\kappa$ is the mean curvature of the interface. The latter work, however, introduces an additional so-called stabilizing function $g(u)=30 u^{2}\left(1-u^{2}\right)$ which is multiplied with the chemical potential, i.e. Rätz et al. consider $g \mu$ instead of $\mu$ in their corresponding version of (1). This factor has been claimed by Guggenberger et al. [20] to remove an inconsistency in the asymptotic derivation which arises because the authors assume that the leading order outer solution is exact i.e. does not require corrections. However, as has been shown previously by Dai and Du [10] for the mobility $\left|1-u^{2}\right|$ and by Lee et al. [34] for the mobility $\left(1-u^{2}\right)_{+}$and solutions that additionally satisfy $|u| \leq 1$, the outer correction problems do indeed have non-trivial contributions. Here, we carry out the systematic matched asymptotic analysis for the mobility $\left(1-u^{2}\right)^{2}$ in the anisotropic case. Our first goal is to show that in the limit $\epsilon \rightarrow 0$ the sharp interface dynamics for anisotropic surface diffusion dewetting is recovered. According to [54], the anisotropic version of the sharpinterface law for surface diffusion is characterized by

$$
v_{n} \propto \partial_{s}\left[D(\gamma) \partial_{s}\left(\left(\gamma+\gamma^{\prime \prime}\right) \kappa\right)\right]
$$

where $v_{n}$ is the normal velocity of the interface, $D(\gamma)$ is an anisotropic phenomenological parameter and $\partial_{s}$ denotes the derivative with respect to the arclength $s$. The sharp interface derivations and numerical simulations presented in this paper are for an isotropic diffusional mobility $m(u)=\left(1-u^{2}\right)^{2}$ similar to the one used in [27]. The sharp interface limit for this particular case leads to $D(\gamma)=\gamma$ in (5). In [49], the authors consider a general mobility $\tilde{m}(u)=\left(1-u^{2}\right)^{2} D(\gamma) / \gamma$ instead of $m(u)$ and therefore their sharp interface derivations results in an expression like (5) with a general anisotropic coefficient function $D(\gamma)$.

Moreover, our analysis takes into account the boundary layers at the substrate and the inner solution at the contact line and derives adequate boundary conditions at triple junctions for the anisotropic Cahn-Hilliard equation. Other studies that deal with the boundary conditions at triple junctions have considered the isotropic Cahn-Hilliard equation [44], or a system of isotropic Cahn-Hilliard equations [19], where the ideas of [4] are adapted in order to show that in the asymptotic limit the boundary condition leads to Young's law at triple junctions [62], i.e.

$$
\sigma_{V S}-\sigma_{F S}=\sigma_{F V} \cos \alpha,
$$

where $\sigma_{V S}, \sigma_{F S}$ and $\sigma_{F V}$ are the interface energy densities describing the interfaces between vapor and substrate, film and substrate, and film and vapor, respectively, $\alpha$ and the equilibrium contact angle. Of particular interest in our study is the technique as well as the geometry presented in [46], in order to study the asymptotic behavior at the contact line for our problem.

As mentioned above, anisotropies in phase-field models and in particular their sharp interface limit $[37,49,18]$ as well as boundary conditions at triple junctions $[44,19,4,46]$ have been discussed in the literature. Here, we carry out the matched asymptotic analysis of the sharp interface limit for a phase-field model with biquadratic degenerate mobility and continuous double well free energy, anisotropic surface energy together with the boundary layer at a substrate and the triple point at the contact line that is required for modeling surface-diffusion driven dewetting of a crystalline film from a substrate.

The paper is organized as follows. First we propose a two-dimensional phase field model for solving the anisotropic surface-diffusion dewetting problem. In section 3 we derive the corresponding sharp-interface limit in the weakly anisotropic case and inside the model domain which 


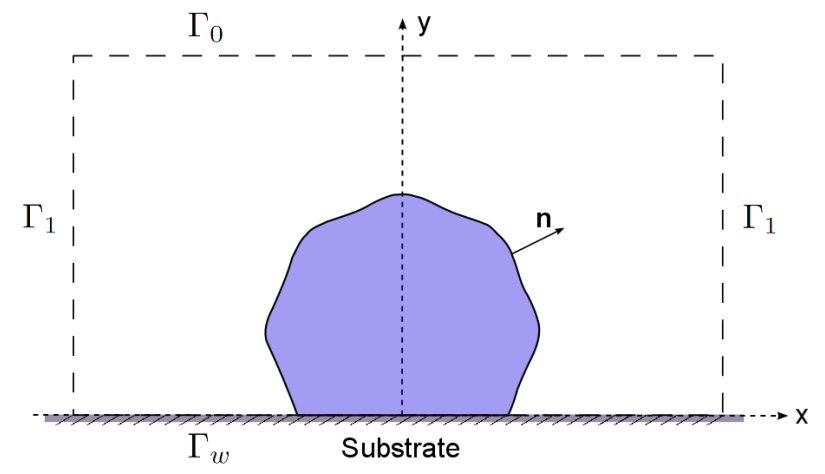

Figure 1: A sketch of the model domain.

confirms the approach of surface diffusion for the present choice of mobility $m$ and free energy $F$. In section 4 we deal with the corresponding boundary condition at the solid boundary and apply an appropriate asymptotic method in order to derive the anisotropic contact angle boundary condition.

\section{Problem formulation}

We consider a one-dimensional film/vapor interface centered around the $x$-axis on a substrate, which we define to be located at $y=0$, and define the domain $\Omega$ to be a two-dimensional rectangular box around this interface, i.e. $\Omega=\left[-L_{x}, L_{x}\right] \times\left[0, L_{y}\right]$, where $L_{x}, L_{y} \in \mathbb{R}^{+}$, with boundary $\partial \Omega=\Gamma_{0} \cup \Gamma_{1} \cup \Gamma_{w}$ (see Fig. 1). The reason for choosing this model domain is that it is easy to handle and adequate for the dewetting of a solid film from a substrate. Then for the phase-field function $u$ as defined in the introduction, the energy functional $W^{\epsilon}$ is generalised, following the approach by Kobayashi [29] and similarly in [60, 55], by introducing an anisotropic gradient contribution to the bulk free energy of the form

$$
f_{F V}(u, \nabla u)=\lambda_{m}\left(F(u)+\frac{\epsilon^{2}}{2} \gamma(\theta)^{2}|\nabla u|^{2}\right),
$$

where $F(u)$ is the homogeneous free energy, $\gamma: \mathbb{R} \rightarrow \mathbb{R}^{+}$is the anisotropic interface energy between film and vapor and $\lambda_{m}$ represents the mixing energy density [23, 63]. In this paper, we will consider the sharp interface limit for the case where the homogeneous free energy is the double well potential

$$
F(u)=\frac{1}{2}\left(1-u^{2}\right)^{2} .
$$

Furthermore, $\gamma$ is a smooth (at least $C^{4}$ ) $2 \pi$-periodic function and $-\pi<\theta \leq \pi$ is the angle between $-\nabla u$ and the $x$-axis. Note that $-\nabla u$ corresponds to the vector which points from the "+" to the "-" phase and may be identified as the outwards pointing normal vector onto the "interface". In order to write $\gamma(\theta)$ in terms of $\nabla u$ we introduce the following common 
generalisation of the arctangent function

$$
\theta=\operatorname{atan} 2\left(u_{y}, u_{x}\right)= \begin{cases}\arctan \frac{u_{y}}{u_{x}} & \text { for } u_{x}>0 \\ \arctan \frac{u_{y}}{u_{x}}+\pi & \text { for } u_{x}<0 \text { and } u_{y} \geq 0 \\ \arctan \frac{u_{y}}{u_{x}}-\pi & \text { for } u_{x}<0 \text { and } u_{y}<0 \\ +\frac{\pi}{2} & \text { for } u_{x}=0 \text { and } u_{y}>0 \\ -\frac{\pi}{2} & \text { for } u_{x}=0 \text { and } u_{y}<0 \\ 0 & \text { for } u_{x}=0 \text { and } u_{y}=0,\end{cases}
$$

so that

$$
\gamma(\theta)=\gamma\left(\operatorname{atan} 2\left(u_{y}, u_{x}\right)\right)
$$

We assume that $\gamma(\theta)=\gamma(-\theta)$ which implies smoothness of $\gamma$ everywhere except for $u_{x}=u_{y}=0$. Note that in this special case all the expressions where $\gamma$ occur become smooth anyway due to multiplication by $u_{x}$ and $u_{y}$. Moreover we will require the interface energy to be only weakly anisotropic, i.e.

$$
\gamma(\theta)+\gamma^{\prime \prime}(\theta)>0
$$

for all $\theta \in[-\pi, \pi]$, to avoid ill-posedness of the resulting evolution equations [16]. To be more precise, if $\gamma^{2}|\nabla u|^{2}$ is not convex then the equation can become backwards parabolic for some initial data $[60,16]$ and in the two-dimensional case, which we consider here, this corresponds to the case if and only if $\gamma(\theta)+\gamma^{\prime \prime}(\theta) \leq 0$, which is referred to as strongly anisotropic. For the wall energy density $f_{w}$, we adhere to [24] and use (4).

We assume that the order parameter $u$ is conserved,

$$
\partial_{t} u=\nabla \cdot \mathbf{j}
$$

and define the mass flux of $u$ to be

$$
\mathbf{j}=m(u) \nabla \mu
$$

where the chemical potential $\mu$ is determined from the first variational derivative of $W^{\epsilon}$ with respect to $u$

$$
\mu=\frac{1}{\lambda_{m}} \frac{\delta W^{\epsilon}}{\delta u}=F^{\prime}(u)-\epsilon^{2} \nabla\left(\gamma \gamma^{\prime}\left(\begin{array}{c}
-u_{y} \\
u_{x}
\end{array}\right)+\gamma^{2} \nabla u\right) .
$$

For $m$, we chose the biquadratic mobility

$$
m(u)=\left(1-u^{2}\right)^{2} .
$$

This is subject to the following boundary conditions

$$
\epsilon \mathbf{n}_{\Omega} \cdot\left[\gamma(\theta) \gamma^{\prime}(\theta)\left(\begin{array}{c}
-u_{y} \\
u_{x}
\end{array}\right)+\gamma(\theta)^{2} \nabla u\right]+\frac{f_{w}^{\prime}}{\lambda_{m}}=0, \quad \mathbf{n}_{\Omega} \cdot(m(u) \nabla \mu)=0,
$$

on $\Gamma_{w}$ and

$$
\mathbf{n}_{\Omega} \cdot \nabla u=0, \quad \mathbf{n}_{\Omega} \cdot(m(u) \nabla \mu)=0,
$$

on $\Gamma_{0} \cup \Gamma_{1}$, where $\mathbf{n}_{\Omega}$ is the outwards pointing normal vector onto $\partial \Omega$. The second of each of these pairs of conditions represents no-flux at the boundary, while the first are the natural boundary conditions for $u$ that arise when calculating the first variation of $W^{\epsilon}$ via integration by parts. 
The aim of this paper is to study the sharp interface limit for the anisotropic phase field model (11) with mobility $m$ defined by (11d) and free energy $F$ defined by (8) on a long time scale $t=O\left(1 / \epsilon^{2}\right)$, using the method of matched asymptotic expansions. Observing that the evolution of the order parameter occurs at an $O\left(1 / \epsilon^{2}\right)$ time scale (see [34]), we suggest to rescale time via $\tau=\epsilon^{2} t$, so that the Cahn-Hilliard equation (11a)-(11c) reads

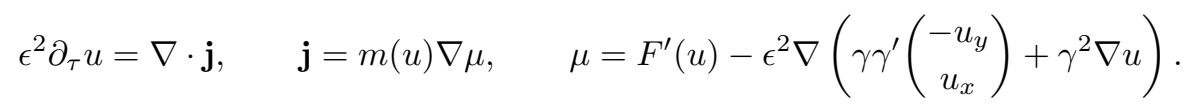

The domain $\Omega$ decomposes into several regions with different scalings: An outer region away from $\Gamma_{w}$ and from the solid-vapor interface $\Gamma$; an inner region at the interface; an inner region at $\Gamma_{w}$ and at an additional free boundary, where the previously mentioned inner regions meet. We now investigate these regions in turn.

\section{Sharp interface asymptotics away from the solid sub- strate}

We first study the asymptotic behavior of the solution in the outer region and the inner interface region away from the solid substrate which is located at $y=0$, i.e. we consider the partial differential equation (12) without the boundary condition (11e). The method of matched asymptotic expansions for anisotropic sharp interface limits has already been applied in [37] and [18] in order to recover the appropriate anisotropic sharp interface form of an anisotropic Allen-Cahn-type equation. In [18] it is in addition pointed out how the analysis has to be modified when considering the Cahn-Hilliard system or the related minimum problem. The Cahn-Hilliard case was also studied in [49] where a connection between sharp interface models for isotropic and anisotropic surface evolution and their diffuse interface counterparts is given. In contrast to our work, [49] as well as [18] also consider different driving forces such as deposition flux and elastic stress in the diffuse interface model, which induce that the evolution in the sharp interface limit is not only driven by surface diffusion. In this section we will present a matched asymptotic analysis for the anisotropic Cahn-Hilliard equation (12) with the aim to recover pure surface diffusion in the sharp interface limit. As shown in [34] this is already in the isotropic case a non-trivial topic and we will exploit this knowledge as well as the particular asymptotic method presented in [34] in order to verify the sharp interface limit in our case.

\subsection{Outer problem}

The equations (12) are already stated in outer variables. For the outer expansions, we will use

$$
\begin{aligned}
u & =u_{0}+\epsilon u_{1}+\epsilon^{2} u_{2} \ldots, \\
\mu & =\mu_{0}+\epsilon \mu_{1}+\epsilon^{2} \mu_{2} \ldots \\
\mathbf{j} & =\mathbf{j}_{0}+\epsilon \mathbf{j}_{1}+\epsilon^{2} \mathbf{j}_{2} \ldots
\end{aligned}
$$

which suggests the following expansions for $m(u)$ and $F(u)$

$$
\begin{aligned}
& m(u)=m\left(u_{0}\right)+\epsilon m^{\prime}\left(u_{0}\right) u_{1}+\epsilon^{2}\left(\frac{1}{2} m^{\prime \prime}\left(u_{0}\right) u_{1}^{2}+m^{\prime}\left(u_{0}\right) u_{2}\right)+O\left(\epsilon^{3}\right) \\
& F^{\prime}(u)=F^{\prime}\left(u_{0}\right)+\epsilon F^{\prime \prime}\left(u_{0}\right) u_{1}+\epsilon^{2}\left(\frac{1}{2} F^{\prime \prime \prime}\left(u_{0}\right) u_{1}^{2}+F^{\prime \prime}\left(u_{0}\right) u_{2}\right)+O\left(\epsilon^{3}\right) .
\end{aligned}
$$




\subsection{Inner problem}

Similar as in $[47,34]$, we define the inner layer in a coordinate system relative to the interface

$$
\mathbf{x}=\mathbf{R}(s, \tau)+\epsilon \rho \mathbf{n}(s, \tau),
$$

and let $U(s, \rho, \tau)=u(x, y, t), M(s, \rho, \tau)=\mu(x, y, t), \mathbf{J}(s, \rho, \tau)=u(x, y, t)$. Here, $\mathbf{R}=\left(r_{1}, r_{2}\right)$ is the position of the interface defined by

$$
u(\mathbf{R}, t)=0,
$$

$s$ is the arclength and $\mathbf{n}=\left(n_{1}, n_{2}\right)^{T}$ is the unit normal to the solid-vapor interface oriented such that it points out of the solid. The orientation of the unit tangent $\mathbf{t}=\left(t_{1}, t_{2}\right)^{T}$ and of the corresponding arclength parametrisation of $R$ are chosen so that $(\mathbf{t}, \mathbf{n})$ forms a right-handed system, $\mathbf{t}=\left(n_{2},-n_{1}\right)^{T}$, thus the solid always lies to the right of the curve. The sign of the curvature $\kappa$ is defined so that the normal and tangent unit vectors satisfy the Frenet-Serret formulae in the form

$$
\partial_{s} \mathbf{t}=-\kappa \mathbf{n}, \quad \partial_{s} \mathbf{n}=\kappa \mathbf{t}
$$

This choice implies that $\kappa>0$ if the curve is convex with respect to the solid. The gradient operator in these curvilinear coordinates reads

$$
\nabla=\mathbf{n} \epsilon^{-1} \partial_{\rho}+\frac{1}{1+\epsilon \rho \kappa} \mathbf{t} \partial_{s}
$$

and for the divergence operator of a vector field $\mathbf{A} \equiv A_{n} \mathbf{n}+A_{s} \mathbf{t}$ we obtain

$$
\nabla \cdot \mathbf{A}=\frac{1}{1+\epsilon \rho \kappa}\left[\epsilon^{-1} \partial_{\rho}\left((1+\epsilon \rho \kappa) A_{n}\right)+\partial_{s}\left(\frac{1}{1+\epsilon \rho \kappa} A_{s}\right)\right] .
$$

For the inner expansions, we will use

$$
\begin{gathered}
U=U_{0}+\epsilon U_{1}+\epsilon^{2} U_{2} \ldots, \\
M=M_{0}+\epsilon M_{1}+\epsilon^{2} M_{2} \ldots
\end{gathered}
$$

Moreover, in view of the last equation in (12), we will apply

$$
F^{\prime}(U)=F^{\prime}\left(U_{0}\right)+\epsilon F^{\prime \prime}\left(U_{0}\right) U_{1} \cdots
$$

and introduce expansions for $\theta$ and $\gamma$ respectively, as these are relevant for the first three orders of the inner problem

$$
\begin{aligned}
& \theta=\theta_{0}+\epsilon \theta_{1}+\epsilon^{2} \theta_{2} \ldots \\
& \gamma=\gamma\left(\theta_{0}\right)+\epsilon \gamma^{\prime}\left(\theta_{0}\right) \theta_{1}+\epsilon^{2}\left(\frac{1}{2} \gamma^{\prime \prime}\left(\theta_{0}\right) \theta_{1}^{2}+\gamma^{\prime}\left(\theta_{0}\right) \theta_{2}\right) \ldots
\end{aligned}
$$

Taylor expanding $\gamma$ in $\epsilon$ around $\epsilon=0$ then reveals the identification

$$
\gamma_{0}=\gamma\left(\theta_{0}\right) \quad \text { and } \quad \gamma_{1}=\gamma^{\prime}\left(\theta_{0}\right) \theta_{1}
$$

The last part in the last equation of (12) can be expanded as

$$
\begin{aligned}
\epsilon^{2}\left(\gamma \gamma^{\prime}\left(\begin{array}{c}
-U_{y} \\
U_{x}
\end{array}\right)\right. & \left.+\gamma^{2} \nabla U\right)=\partial_{\rho}\left(\gamma_{0}^{2} \partial_{\rho} U_{0}\right) \\
& +\epsilon\left(\mathbf{t} \partial_{s}\left(-\gamma_{0} \gamma_{0}^{\prime} \mathbf{t} \partial_{\rho} U_{0}+\gamma_{0}^{2} \mathbf{n} \partial_{\rho} U_{0}\right)+\mathbf{n} \partial_{\rho}\left(\gamma_{0} \gamma_{0}^{\prime} \mathbf{n} \partial_{s} U_{0}+\gamma_{0}^{2} \mathbf{t} \partial_{s} U_{0}\right)\right. \\
& \left.+\mathbf{n} \partial_{\rho}\left(-\left(\gamma_{1} \gamma_{0}^{\prime}+\gamma_{0} \gamma_{1}^{\prime}\right) \mathbf{t} \partial_{\rho} U_{0}-\gamma_{0} \gamma_{0}^{\prime} \mathbf{t} \partial_{\rho} U_{1}+2 \gamma_{0} \gamma_{1} \mathbf{n} \partial_{\rho} U_{0}+\gamma_{0}^{2} \mathbf{n} \partial_{\rho} U_{1}\right)\right) \ldots
\end{aligned}
$$


For further analysis it will prove useful to calculate $\gamma_{0}, \gamma_{1}$ or $\theta_{0}, \theta_{1}$ explicitly in view of inner coordinates, but since the corresponding calculation is long and technical we skip it at this point and refer the reader to Appendix A for a detailed derivation. The result is the following representation of $\theta_{0}$ and $\theta_{1}$

$$
\theta_{0}= \begin{cases}\operatorname{atan} 2\left(n_{2}, n_{1}\right) & \text { for } n_{1} \neq 0 \\ \pm \frac{\pi}{2} & \text { for } n_{1}=0\end{cases}
$$

and

$$
\theta_{1}=\left\{\begin{aligned}
-\frac{U_{s}}{U_{\rho}} & \text { for } n_{1} \neq 0 \\
0 & \text { for } n_{1}=0
\end{aligned}\right.
$$

Hence, the leading order of $\theta$ and therefore also of $\gamma($ see $(22))$ are independent of $\rho$.

Applying the inner expansions in (12) we find that, the first two equations combined become

$$
\epsilon^{2} \partial_{\tau} U-\epsilon v_{n} \partial_{\rho} U=\nabla(m(U) \nabla M)
$$

with $v_{n}=\partial_{\tau} \mathbf{R} \cdot \mathbf{n}$ and where

$$
\begin{aligned}
\nabla \cdot(m(U) \nabla)= & \epsilon^{-2} \partial_{\rho} m\left(U_{0}\right) \partial_{\rho}+\epsilon^{-1}\left[\partial_{\rho}\left(\kappa \rho m\left(U_{0}\right)+m^{\prime}\left(U_{0}\right) U_{1}\right) \partial_{\rho}-\kappa \rho \partial_{\rho} m\left(U_{0}\right) \partial_{\rho}\right] \\
& +\left[\kappa^{2} \rho^{2} \partial_{\rho} m\left(U_{0}\right) \partial_{\rho}-\kappa \rho \partial_{\rho}\left(\kappa \rho m\left(U_{0}\right)+m^{\prime}\left(U_{0}\right) U_{1}\right) \partial_{\rho}\right. \\
& \left.+\partial_{\rho}\left(\kappa \rho m^{\prime}\left(U_{0}\right) U_{1}+\frac{1}{2} m^{\prime \prime}\left(U_{0}\right) U_{1}^{2}+m^{\prime}\left(U_{0}\right) U_{2}\right) \partial_{\rho}+\partial_{s} m\left(U_{0}\right) \partial_{s}\right]+O(\epsilon) .
\end{aligned}
$$

All together we obtain

$$
\begin{aligned}
\epsilon^{4} \partial_{\tau} U- & \epsilon^{3} v_{n} \partial_{\rho} U=\partial_{\rho}\left(m\left(U_{0}\right) \partial_{\rho} M_{0}\right) \\
+ & \epsilon\left[\partial_{\rho}\left(m\left(U_{0}\right) \partial_{\rho} M_{1}\right)+\partial_{\rho}\left(\left(\kappa \rho m\left(U_{0}\right)+m^{\prime}\left(U_{0}\right) U_{1}\right) \partial_{\rho} M_{0}\right)-\kappa \rho \partial_{\rho}\left(m\left(U_{0}\right) \partial_{\rho} M_{0}\right)\right] \\
+ & \epsilon^{2}\left[\partial_{\rho}\left(m\left(U_{0}\right) \partial_{\rho} M_{2}\right)+\partial_{\rho}\left(\left(\kappa \rho m\left(U_{0}\right)+m^{\prime}\left(U_{0}\right) U_{1}\right) \partial_{\rho} M_{1}\right)-\kappa \rho \partial_{\rho}\left(m\left(U_{0}\right) \partial_{\rho} M_{1}\right)\right. \\
& \quad+\kappa^{2} \rho^{2} \partial_{\rho}\left(m\left(U_{0}\right) \partial_{\rho} M_{0}\right)-\kappa \rho \partial_{\rho}\left(\left(\kappa \rho m\left(U_{0}\right)+M^{\prime}\left(U_{0}\right) U_{1}\right) \partial_{\rho} M_{0}\right) \\
& \left.+\partial_{\rho}\left(\left(\kappa \rho m^{\prime}\left(U_{0}\right) U_{1}+\frac{1}{2} m^{\prime \prime}\left(U_{0}\right) U_{1}^{2}+m^{\prime}\left(U_{0}\right) U_{2}\right) \partial_{\rho} M_{0}\right)+\partial_{s}\left(m\left(U_{0}\right) \partial_{s} M_{0}\right)\right] \\
+ & O\left(\epsilon^{3}\right) .
\end{aligned}
$$

Finally we motivate the inner asymptotic expansions for $\mathbf{J}$. Taking only the first equation in (12) we have

$$
\epsilon^{2} \partial_{\tau} U-\epsilon v_{n} \partial_{\rho} U=\frac{1}{1+\epsilon \rho \kappa}\left[\epsilon^{-1} \partial_{\rho}((1+\epsilon \rho \kappa) \mathbf{n} \cdot \mathbf{J})+\partial_{s}\left(\frac{1}{1+\epsilon \rho \kappa} \mathbf{s} \cdot \mathbf{J}\right)\right]
$$

which reveals that the normal component $J_{n}=\mathbf{n} \cdot \mathbf{J}$ is the dominant contribution of $\mathbf{J}$ in the 
inner evolution equation. This normal component $J_{n}$ can be expanded as

$$
\begin{aligned}
J_{n}= & \frac{m(U)}{\epsilon} \partial_{\rho} M \\
= & \epsilon^{-1} m\left(U_{0}\right) \partial_{\rho} M_{0}+m^{\prime}\left(U_{0}\right) U_{1} \partial_{\rho} M_{0}+m\left(U_{0}\right) \partial_{\rho} M_{1} \\
& +\epsilon\left[m\left(U_{0}\right) \partial_{\rho} M_{2}+m^{\prime}\left(U_{0}\right) U_{1} \partial_{\rho} M_{1}+m^{\prime}\left(U_{0}\right) U_{2} \partial_{\rho} M_{0}+\frac{1}{2} m^{\prime \prime}\left(U_{0}\right) U_{1}^{2} \partial_{\rho} M_{0}\right] \\
& +\epsilon^{2}\left[m\left(U_{0}\right) \partial_{\rho} M_{3}+m^{\prime}\left(U_{0}\right) U_{1} \partial_{\rho} M_{2}+\left(m^{\prime}\left(U_{0}\right) U_{2}+\frac{1}{2} m^{\prime \prime}\left(U_{0}\right) U_{1}^{2}\right) \partial_{\rho} M_{1}\right. \\
& \left.\quad+\left(m^{\prime}\left(U_{0}\right) U_{3}+m^{\prime \prime}\left(U_{0}\right) U_{1} U_{2}+\frac{1}{6} m^{\prime \prime \prime}\left(U_{0}\right) U_{1}^{3}\right) \partial_{\rho} M_{0}\right]+O\left(\epsilon^{3}\right) .
\end{aligned}
$$

which motivates the following inner expansions for $\mathbf{J}$

$$
\mathbf{J}=\epsilon^{-1} \mathbf{J}_{-1}+\mathbf{J}_{0}+\epsilon \mathbf{J}_{1}+\epsilon^{2} \mathbf{J}_{2} \cdots
$$

Note that in the following we will refer to $J_{n, i}$ as the $O\left(\epsilon^{i}\right)$ term of $J_{n}$.

\subsection{Solutions with $|u| \leq 1$}

In this paper, we focus on solutions of the phase field model with $|u| \leq 1$, for which existence has been proved for the standard (isotropic) Cahn-Hilliard equation with degenerate mobilities in [17]. On the other hand, an existence proof for the isotropic Cahn-Hilliard equation that does not impose $|u| \leq 1$ has been given in [11], and numerical solutions with regions where $|u|>1$ have been discussed in $[34,12]$. These results are not in contradiction, as uniqueness cannot in general be expected for degenerate PDEs. It is therefore reasonable to expect that these two types of solutions also exist for the anisotropic model (12). Interestingly, for the mobility $m(u)=\left|1-u^{2}\right|$ (which has simple zeros at $u= \pm 1$ ), the two types of solutions lead to different sharp interface limits $[34,9]$, but even in the case of the biquadratic mobility $m(u)=\left|1-u^{2}\right|^{2}$ that we use here, there are technical differences between the two cases (resulting from the introduction of $\chi$, see below), so we have to make a choice before we carry out the asymptotic analysis. We have chosen the case where $u$ remains in the range $|u| \leq 1$. This type of solutions has been widely considered in the literature [17], where it is often argued to be physically desirable that the phase-field variables do not exceed the values $u= \pm 1$, since these correspond to the pure phases.

The sharp interface limit describes the long-time asymptotic quasi-stationary behaviour of the interface. The first two orders are, in fact, stationary solutions, both in inner and outer variables. It turns out that solutions of the stationary Cahn-Hillard equation

$$
\mu=\epsilon^{2} \Delta u-F^{\prime}(u)=\text { const }
$$

near a non-flat interface typically have solutions with values that do not remain in the range $|u| \leq 1$, see $[10,34,12]$. This can be seen particularly easily for radially symmetric solutions of the stationary Cahn-Hillard equation [43] which usually exceed $|u|=1$ in a region around the origin until close to the (diffuse) interface [34]. This raises the question how we can carry out a quasi-stationary limit if the solutions of the stationary Cahn-Hilliard equation exceed the permissible range $|u| \leq 1$.

The apparent contradiction can be resolved by observing that the degenerate mobility (12) increases the range of candidates for stationary solutions, which now can be pieced together from solutions of (31) and parts where $u=1$ or $u=-1$. Indeed, numerical experiments carried out 
in [34] suggest that solutions of the Cahn-Hilliard equation with degenerate mobility converge to a profile for $u$ where $u=1$ along a curve on the convex side of the interface. We follow the example in [34] (and also refer to this article for an expanded discussion on the introduction of $\chi$ ) and assume that $u$ and $\mathbf{j}$ satisfy

$$
u=1, \quad \mathbf{j} \cdot \mathbf{n}_{\chi}=0, \quad \nabla u \cdot \mathbf{n}_{\chi}=0,
$$

at $\mathbf{x}=\chi$, where $\mathbf{n}_{\chi}$ is the normal to $\chi$. We thus introduce a free boundary at $\chi$, and avoid having to analyse the solution beyond the point $\mathbf{x}=\chi$ where $u$ reaches \pm 1 .

Now let $\rho=-\omega(s, \tau)$ be the position of $\chi$ in inner (i.e. $\rho$-) coordinates. To take (32) into account, it is useful to introduce a second inner layer at $\chi$ in addition to the layer at $\mathbf{x}=\mathbf{R}$. This will allow us to introduce the conditions (32) by enforcing them on the solution of the additional layer and then matching this layer to the inner layer at $\mathbf{x}=\mathbf{R}$. For this purpose introduce shifted inner coordinates, centered at $\chi$, via

$$
z=\rho+\omega(s, \tau)
$$

so that the transformation between the new inner and the outer coordinates is, using (14),

$$
\mathbf{x}=\mathbf{R}(s, \tau)+\epsilon(z-\omega(s, \tau)) \mathbf{n}(s, \tau) .
$$

The corresponding inner expansions may then be written as

$$
\begin{aligned}
\bar{U} & =1+\epsilon \bar{U}_{1}+\epsilon^{2} \bar{U}_{2} \ldots, \\
\bar{M} & =\bar{M}_{0}+\epsilon \bar{M}_{1}+\epsilon^{2} \bar{M}_{2} \ldots, \\
\overline{\mathbf{J}} & =\epsilon^{-1} \overline{\mathbf{J}}_{-1}+\overline{\mathbf{J}}_{0}+\epsilon \overline{\mathbf{J}}_{1}+\epsilon^{2} \overline{\mathbf{J}}_{2} \ldots
\end{aligned}
$$

and the boundary conditions (32) become

$$
\bar{U}(0)=1, \quad J_{z}=0, \quad \partial_{z} \bar{U}(0)=0,
$$

where $J_{z}$ denotes the $z$-component of $\overline{\mathbf{J}}$. Note that since the position of the two inner layers depends also on $\epsilon$, the positions $\omega$ and $\mathbf{R}$ actually need to be expanded in terms of $\epsilon$ as well. However, since we are only interested in the leading order behaviour of the interface we use $\omega$ and $\mathbf{R}$ and their leading order contributions interchangeably. We now solve and match the outer and inner problems order by order.

\subsection{Matching}

\section{Leading order}

For the leading order outer problem we obtain

$$
0=\nabla \cdot \mathbf{j}_{0}, \quad \mathbf{j}_{0}=m\left(u_{0}\right) \nabla \mu_{0}, \quad \mu_{0}=F^{\prime}\left(u_{0}\right),
$$

and the corresponding boundary conditions are $\mathbf{n}_{\Omega} \cdot \nabla u=0$ and $\mathbf{n}_{\Omega} \cdot \mathbf{j}_{0}=0$. Since we suppose that the "-" phase is outside the solid film, we conclude that

$$
u_{0}=-1, \quad \mu_{0}=0 .
$$

The leading order inner expansion reads

$$
\begin{aligned}
\partial_{\rho}\left(m\left(U_{0}\right) \partial_{\rho} M_{0}\right) & =0, \\
F^{\prime}\left(U_{0}\right)-\partial_{\rho}\left(\gamma_{0}^{2} \partial_{\rho} U_{0}\right) & =M_{0} .
\end{aligned}
$$


Integrating once in $\rho$, we obtain

$$
m\left(U_{0}\right) \partial_{\rho} M_{0}=a_{1}(s, \tau)
$$

From the matching conditions we require

$$
\lim _{\rho \rightarrow \infty} U_{0}(\rho)=-1
$$

which implies $a_{1} \equiv 0$ and therefore also $M_{0}=0$. Moreover, from (24) we know that $\theta_{0}$ is constant in $\rho$, which leads to

$$
2\left(U_{0}^{3}-U_{0}\right)-\gamma_{0}^{2} \partial_{\rho \rho} U_{0}=0
$$

and, by applying the phase condition $U_{0}(0)=0$ (obtained from (15)), consequently

$$
U_{0}=-\tanh \left(\frac{1}{\gamma_{0}} \rho\right) \text {. }
$$

Using $M_{0}=0$ we also conclude that

$$
J_{n,-1}=0 \text {. }
$$

Finally it is easily seen, that from the inner expansions about $\chi$ we get

$$
\bar{U}_{0}=1, \quad \bar{M}_{0}=0, \quad \bar{J}_{n,-1}=0 .
$$

\section{$\boldsymbol{O}(\epsilon)$ correction}

The first two parts of the outer $O(\epsilon)$ correction problem for (12) are trivial, since $\mu_{0}=0$ and $m\left(u_{0}\right)=0$ and consequently

$$
\mathbf{j}_{1}=0 \text {. }
$$

The last equation becomes

$$
\mu_{1}=F^{\prime \prime}\left(u_{0}\right) u_{1}=4 u_{1},
$$

which we need to match to $M_{1}$ in the following. As $M_{0}=0$ we obtain for the first equation of the inner correction problem

$$
\partial_{\rho}\left(m\left(U_{0}\right) \partial_{\rho} M_{1}\right)=0
$$

such that $m\left(U_{0}\right) \partial_{\rho} M_{1}$ is constant in $\rho$. Comparison with (29) then reveals that (47) corresponds to the normal flux term $J_{n, 0}$, which has to match with $j_{0}$ and consequently is zero. Thus $M_{1}$ does not depend on $\rho$.

Applying curvilinear coordinates the equation for $M_{1}$ reads

$$
\begin{aligned}
M_{1}= & F^{\prime \prime}\left(U_{0}\right) U_{1}-\left(\mathbf{t} \partial_{s}\left(-\gamma_{0} \gamma_{0}^{\prime} \mathbf{t} \partial_{\rho} U_{0}+\gamma_{0}^{2} \mathbf{n} \partial_{\rho} U_{0}\right)+\mathbf{n} \partial_{\rho}\left(\gamma_{0} \gamma_{0}^{\prime} \mathbf{n} \partial_{s} U_{0}+\gamma_{0}^{2} \mathbf{t} \partial_{s} U_{0}\right)\right. \\
& \left.+\mathbf{n} \partial_{\rho}\left(-\left(\gamma_{1} \gamma_{0}^{\prime}+\gamma_{0} \gamma_{1}^{\prime}\right) \mathbf{t} \partial_{\rho} U_{0}-\gamma_{0} \gamma_{0}^{\prime} \mathbf{t} \partial_{\rho} U_{1}+2 \gamma_{0} \gamma_{1} \mathbf{n} \partial_{\rho} U_{0}+\gamma_{0}^{2} \mathbf{n} \partial_{\rho} U_{1}\right)\right)
\end{aligned}
$$

Exploiting that $\gamma_{0}, \mathbf{n}$ and $\mathbf{t}$ do not depend on $\rho$, applying the two-dimensional Frenet-Serret formulae (16), i.e.

$$
\partial_{s} \mathbf{t}=-\kappa \mathbf{n}, \quad \partial_{s} \mathbf{n}=\kappa \mathbf{t},
$$

and using the $\rho$-independence of $\theta_{0}$ (see (24)) in order to calculate $\partial_{s} \gamma_{0}$, equation (48) becomes

$$
M_{1}=F^{\prime \prime}\left(U_{0}\right) U_{1}-\kappa\left(\gamma_{0}^{\prime \prime}+\gamma_{0}\right) \gamma_{0} \partial_{\rho} U_{0}+\kappa \gamma_{0}^{\prime 2} \partial_{\rho} U_{0}+2 \kappa \gamma_{0}^{\prime 2} \rho \partial_{\rho \rho} U_{0}-\gamma_{0}^{2} \partial_{\rho \rho} U_{1}
$$


Note that we also exploited the particular representation of $\theta_{1}$ in $\gamma_{1}=\gamma_{0}^{\prime} \theta_{1}$, which is

$$
\theta_{1} \sim-\frac{\gamma_{0}^{\prime}}{\gamma_{0}} \rho \kappa
$$

and whose derivation can be found in Appendix B. From (49) we then obtain the ordinary differential equation

$$
\gamma_{0}^{2} \partial_{\rho \rho} U_{1}-2\left(3 U_{0}^{2}-1\right) U_{1}=-\kappa c_{1} \gamma_{0} \partial_{\rho} U_{0}+\kappa c_{2} \gamma_{0} \partial_{\rho} U_{0}+2 \kappa c_{2} \gamma_{0} \rho \partial_{\rho \rho} U_{0}-M_{1},
$$

where we substituted $c_{1}:=\gamma_{0}^{\prime \prime}+\gamma_{0}$ and $c_{2}:=\gamma_{0}^{\prime 2} / \gamma_{0}$. Then the general solution of (51) is given by

$$
\begin{aligned}
U_{1}= & C_{1} \operatorname{sech}^{2}\left(\frac{\rho}{\gamma_{0}}\right)+C_{2} \operatorname{sech}^{2}\left(\frac{\rho}{\gamma_{0}}\right)\left(\frac{3 \rho}{8 \gamma_{0}}+\frac{1}{4} \sinh \left(\frac{2 \rho}{\gamma_{0}}\right)+\frac{1}{32} \sinh \left(\frac{4 \rho}{\gamma_{0}}\right)\right) \\
& +\frac{1}{8}\left(2 c_{1} \kappa-M_{1}\right)+\frac{1}{48}\left(2 c_{1} \kappa-3 M_{1}\right)\left(2 \cosh \left(\frac{2 \rho}{\gamma_{2}}\right)-5 \operatorname{sech}^{2}\left(\frac{\rho}{\gamma_{0}}\right)\right) \\
& -\frac{1}{2} c_{2} \kappa\left(\frac{\rho}{\gamma_{0}}\right)^{2} \operatorname{sech}^{2}\left(\frac{\rho}{\gamma_{0}}\right),
\end{aligned}
$$

and including the interface condition $U_{1}(0)=0$ and boundedness as $\rho \rightarrow \infty$ to match with the outer solution, the two constants are given by

$$
C_{1}=-\frac{1}{16}\left(M_{1}+2 c_{1} \kappa\right), \quad C_{2}=\frac{1}{3}\left(3 M_{1}-2 c_{1} \kappa\right) .
$$

Finally for the inner layer about $\chi$, according to $\bar{U}_{0} \equiv 1$, we obtain $F^{\prime \prime}\left(\bar{U}_{0}\right)=4$ and $\partial_{\rho} \bar{U}_{0}=0$. Inserting this into the analogous equation to (49), we obtain

$$
M_{1}=4 \bar{U}_{1}-\gamma_{0}^{2} \partial_{z z} \bar{U}_{1},
$$

with initial conditions

$$
\bar{U}_{1}(0)=\bar{U}_{1}^{\prime}(0)=0 .
$$

The general solution of (54) is given by

$$
\bar{U}_{1}=A \exp (2 z)+B \exp (-2 z)+\frac{M_{1}}{4}
$$

and substituting the initial conditions (55) we arrive at

$$
\bar{U}_{1}=\frac{M_{1}}{4}\left(1-\cosh \left(\frac{2 z}{\gamma_{0}}\right)\right) .
$$

\subsubsection{Matching of exponential terms}

We will now match the two interior layers. We first observe that on the one hand, from the definition of $\omega(s, \tau)$ in the paragraph before equation (33), we have that $U(s, \omega, \tau)=1$ and $U^{\prime}(s, \omega, \tau)=0$. On the other hand, for $\epsilon \rightarrow 0$, we also have that $U \rightarrow U_{0}=-\tanh \left(\rho / \gamma_{0}\right)<1$ which suggests to assume $\omega(s, \tau) \rightarrow \infty$ for $\epsilon \rightarrow 0$. Matching of the inner expansions therefore involves exponential terms with large negative arguments $\rho$, which we deal with in the spirit of Lange [32]. The corresponding method entails to explicitly match the exponentially growing and decaying terms in the expansion. Note that this method was also considered in Lee et al. [34] 
and has been generalized to partial differential equations of higher (fourth and sixth) order in [30]. The solution centered at the interface is expanded at $\rho \rightarrow-\infty$ and the result written and re-expanded in terms of $z=\rho+\omega(s, \tau)$. The solution for the layer around the free boundary $\xi$ is directly expanded in terms of $z \rightarrow \infty$ and then the terms are matched between the two expansions.

Expanding $U_{0}$ and $U_{1}$ for $\rho \rightarrow-\infty$ and substituting $\rho=z-\omega$ gives

$$
\begin{aligned}
U_{0}= & 1-\underbrace{2 e^{-\frac{2}{\gamma_{0}} \omega} e^{\frac{2}{\gamma_{0}} z}}_{\mathrm{A}}+O\left(e^{4 z}\right), \\
U_{1}= & \underbrace{\frac{1}{24}\left(2 c_{1} \kappa-3 M_{1}\right) e^{\frac{2}{\gamma_{0}} \omega} e^{-\frac{2}{\gamma_{0}} z}+\underbrace{\frac{1}{2}\left(c_{1} \kappa-M_{1}\right)}_{\mathrm{C}}}_{\mathrm{B}} \\
& +\underbrace{\left[\left(\frac{7 M_{1}}{4}-\frac{11 c_{1} \kappa}{6}\right)+\left(\frac{3 M_{1}}{2}-c_{1} \kappa\right) \frac{z-\omega}{\gamma_{0}}-2 c_{2} \kappa\left(\frac{z-\omega}{\gamma_{0}}\right)^{2}\right] e^{-\frac{2}{\gamma_{0}} \omega} e^{\frac{2}{\gamma_{0}} z}}_{\mathrm{D}} \\
& +O\left(e^{4 z}\right) .
\end{aligned}
$$

The inner expansion about the free boundary can be rewritten as

$$
\bar{U}=1+\underbrace{\frac{\epsilon M_{1}}{4}}_{\mathrm{E}}-\underbrace{\frac{\epsilon M_{1}}{8} e^{\frac{2}{\gamma_{0}}} z}_{\mathrm{F}}-\underbrace{\frac{\epsilon M_{1}}{8} e^{-\frac{2}{\gamma_{0}} z}}_{\mathrm{G}}+O\left(\epsilon^{2}\right) .
$$

The terms of the same dependence on $z$ and $\epsilon$ are now matched in the expansions (58) and (59). We first observe that the constant terms at $O(1)$ are already matched. Matching $\epsilon \mathrm{C}$ and E yields

$$
\frac{M_{1}}{4}=\frac{1}{2}\left(c_{1} \kappa-M_{1}\right)
$$

where $c_{1}=\gamma_{0}^{\prime \prime}+\gamma_{0}>0$, thus

$$
M_{1}=\frac{2}{3}\left(\gamma_{0}^{\prime \prime}+\gamma_{0}\right) \kappa
$$

Applying (61) in $\mathrm{B}$ reveals $\mathrm{B}=0$. Matching term $A$ and $F$ we arrive at

$$
2 e^{-\frac{2}{\gamma_{0}} \omega}=\frac{\epsilon}{12}\left(\gamma_{0}^{\prime \prime}+\gamma_{0}\right) \kappa,
$$

which we solve for $\omega$ giving

$$
\omega=\frac{\gamma_{0}}{2} \log \left(\frac{24}{\epsilon\left(\gamma_{0}^{\prime \prime}+\gamma_{0}\right)}\right)
$$

This asymptotic analysis shows that without the contact line $\chi$, i.e. if we were to consider only one inner layer about the interface and would match to the outer solution of the "+ " phase via $U^{\prime}=0$ for $\rho \rightarrow-\infty$, we would expect an outer solution (of the "+" phase) which tends to a value of 1 plus a positive $O(\epsilon)$ term, i.e. $u=1+c \epsilon\left(\gamma_{0}^{\prime \prime}+\gamma_{0}\right) \kappa+O\left(\epsilon^{2}\right)$, where $c>0$. This can be seen by matching (46) to (61). This solution intersects $u=1$ at a distance $\omega=O(\log (1 / \epsilon))$ from the interface, which is large but tends to zero in the outer variable. The assumption $|u| \leq 1$ thus implies that (32) has to be satisfied in the inner variable but since $\omega$ depends, though only logarithmically, on $\epsilon$, this involves exponentially re-expanding the inner solution.

Note that at this stage, it is obvious that the matching is not yet complete to $O(\epsilon)$, as the terms in (58) and (59), or to be more precise $\epsilon \mathrm{D}$ and $G$, are non-zero and lack counterparts in the 
outer expansion. Applying (63) in $\epsilon D$ reveals that $\epsilon D$ is of the form $\epsilon^{2} e^{-\frac{2}{\gamma_{0}} z}$ which shows that the matching at this point can only be resolved by considering the next higher order solutions $U_{2}$ and $\bar{U}_{2}$. However, since this consideration does not influence the following asymptotic analysis, we omit it at this point.

\section{$\boldsymbol{O}\left(\boldsymbol{\epsilon}^{2}\right)$ correction}

Since $m^{\prime}\left(u_{0}\right)=0$ we obtain for the outer correction problem

$$
\mathbf{n} \cdot \mathbf{j}_{2}=0
$$

and again the first two parts of (12) are automatically satisfied. In view of the outer expansions of $F^{\prime}(u)$ and recalling that $u_{0}=-1$, the last part requires

$$
\mu_{2}=\frac{1}{2} F^{\prime \prime \prime}\left(u_{0}\right) u_{1}^{2}+F^{\prime \prime}\left(u_{0}\right) u_{2}
$$

where $F^{\prime \prime \prime}\left(u_{0}\right)=-12$ and $F^{\prime \prime}\left(u_{0}\right)=4$.

Considering the inner correction problem and recalling that $M_{0}, M_{1}$ are independent of $\rho$ we obtain for the first part of (12)

$$
\partial_{\rho}\left(m\left(U_{0}\right) \partial_{\rho} M_{2}\right)=0,
$$

thus $m\left(U_{0}\right) \partial_{\rho} M_{2}$ is constant in $\rho$ and since we can identify this expression via (29) as $J_{n, 1}$ which has to match with $\mathbf{n} \cdot \mathbf{j}_{1}$ we find that

$$
J_{n, 1}=m\left(U_{0}\right) \partial_{\rho} M_{2}=0 .
$$

Therefore, $M_{2}$ is independent of $\rho$.

\section{$O\left(\epsilon^{3}\right)$ correction}

Consider the inner correction problem at this point. Since we have $m^{\prime}\left(U_{0}\right)=m^{\prime \prime}\left(U_{0}\right)=0$ we obtain from (29) that

$$
J_{n, 2}=m\left(U_{0}\right) \partial_{\rho} M_{3} .
$$

For $\rho \rightarrow-\infty$ the left hand side has to match with $\bar{J}_{n, 2}$ and the right hand side with $m(1) \partial_{\rho} \bar{M}_{3}$, but since $\bar{J}_{n, 2}=0$ we immediately obtain

$$
\lim _{\rho \rightarrow-\infty} J_{n, 2}=\lim _{\rho \rightarrow-\infty} m\left(U_{0}\right) \partial_{\rho} M_{3}=0 .
$$

Moreover, $J_{n, 2}$ also matches with $\mathbf{n} \cdot \mathbf{j}_{2}=0$ for $\rho \rightarrow \infty$. Considering the last part of the correction problem for (12) and exploiting that $M_{0}, M_{1}$ and $M_{2}$ are independent of $\rho$ we find

$$
\begin{aligned}
-v_{n} \partial_{\rho} U_{0} & =\partial_{\rho}\left(m\left(U_{0}\right) \partial_{\rho} M_{3}\right)+\partial_{s}\left(m\left(U_{0}\right) \partial_{s} M_{1}\right) \\
& =\partial_{\rho}\left(m\left(U_{0}\right) \partial_{\rho} M_{3}\right)+\frac{2}{3} \partial_{s}\left(m\left(U_{0}\right) \partial_{s}\left(c_{1} \kappa\right)\right) .
\end{aligned}
$$

An integration over $(-\infty, \infty)$ then yields

$$
\begin{aligned}
v_{n} & =\frac{1}{3} \partial_{s}\left[\left(\int_{-\infty}^{\infty} m\left(U_{0}\right) d \rho\right) \partial_{s}\left(c_{1} \kappa\right)\right] \\
& =\left(\frac{2}{3}\right)^{2} \partial_{s}\left[\gamma_{0} \partial_{s}\left(c_{1} \kappa\right)\right] .
\end{aligned}
$$


Finally we obtain the sharp interface problem which correctly describes the anisotropic evolution due to surface diffusion (compare to (5) in the introduction)

$$
\begin{aligned}
\mu_{1} & =\frac{2}{3}\left(\gamma_{0}+\gamma_{0}^{\prime \prime}\right) \kappa \\
v_{n} & =\left(\frac{2}{3}\right)^{2} \partial_{s}\left[\gamma_{0} \partial_{s}\left(\left(\gamma_{0}+\gamma_{0}^{\prime \prime}\right) \kappa\right)\right]
\end{aligned}
$$

on $\chi_{0}$.

\section{Sharp interface dynamics on solid boundaries}

We now focus our studies on the behavior of the anisotropic Cahn-Hilliard equation (12) in a local domain around the contact point $\left(x_{c}, 0\right)$ with boundary condition (11e). The more general topic of boundary conditions at triple junctions has already been studied by $[4,18]$ for the Allen-Cahn equation and in [44] for an Allen-Cahn/Cahn-Hilliard system where in both cases the surface energies are assumed to be isotropic which leads to Young's law in the sharp interface limit. Another work by Owen et al. [46] considers the boundary conditions for an Allen-Cahn gradient flow on a solid substrate where the corresponding geometry turns out to be suitable for our problem.

Motivated by [46] we study the behaviour of $u$ in a box around the contact point $\left(x_{c}, 0\right)$. Introducing a boundary layer and an interior layer which imply corresponding matching conditions, we will show that the leading order system of (12) with boundary condition (11e) leads to a contact angle boundary condition, which is referred to as the Young-Herring condition in the literature $[3,38]$. The subsequent analysis is given for the left contact point but carries over correspondingly to the right contact point.

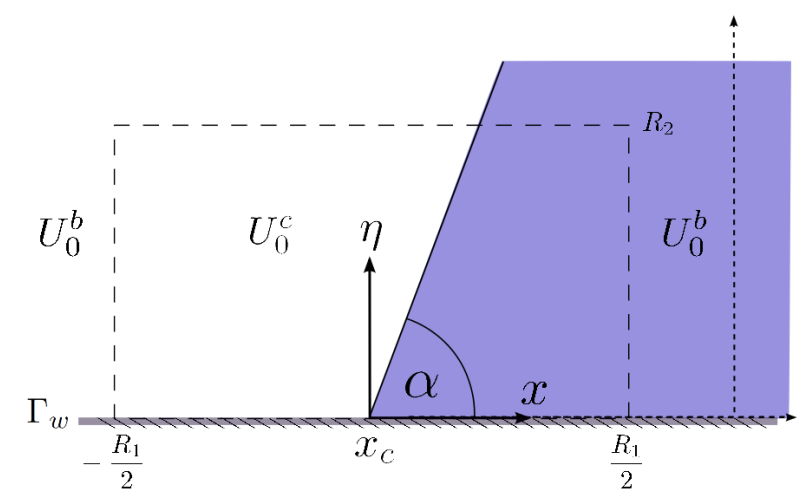

Figure 2: A sketch of the local domain.

Boundary layer near $\Gamma_{w}$ We first introduce the inner variable near $\Gamma_{w}$ via

$$
\eta=\frac{y}{\epsilon}
$$

see Fig. 2, and correspondingly $U^{b}, M^{b}, \gamma^{b}$ and $\theta^{b}$. We expand $U^{b}(x, \eta)$ and $M^{b}$ into

$$
\begin{aligned}
U^{b} & =U_{0}^{b}+\epsilon U_{1}^{b}+\epsilon^{2} U_{2}^{b} \cdots, \\
M^{b} & =M_{0}^{b}+\epsilon M_{1}^{b}+\epsilon^{2} M_{2}^{b} \cdots
\end{aligned}
$$


Moreover we find for $\gamma^{b}$ the expansion

$$
\begin{array}{rlrl}
\gamma^{b} & =\gamma\left(\theta_{0}^{b}\right) & +\epsilon \gamma^{\prime}\left(\theta_{0}^{b}\right) \theta_{1}^{b}+\ldots \\
& =: \quad \gamma_{0}^{b} & +\epsilon \gamma_{1}^{b} & +\ldots
\end{array}
$$

where

$$
\theta_{0}^{b}=\lim _{\epsilon \rightarrow 0} \operatorname{atan} 2\left(\partial_{\eta} U_{0}^{b}, \epsilon \partial_{x} U_{0}^{b}\right)=\left\{\begin{aligned}
+\frac{\pi}{2} & \text { for } \partial_{\eta} U_{0}^{b}>0 \\
-\frac{\pi}{2} & \text { for } \partial_{\eta} U_{0}^{b}<0 \\
0 & \text { for } \partial_{\eta} U_{0}^{b}=0
\end{aligned}\right.
$$

such that $\gamma_{0}^{b}=\gamma\left(\theta_{0}^{b}\right)=\gamma\left( \pm \frac{\pi}{2}\right)$ is constant. The leading order problem of (12) then reads

$$
\begin{aligned}
0 & =\partial_{\eta}\left(m\left(U_{0}^{b}\right) \partial_{\eta} M_{0}^{b}\right), \\
M_{0}^{b} & =F^{\prime}\left(U_{0}^{b}\right)-\partial_{\eta}\left(\left(\gamma_{0}^{b}\right)^{2} \partial_{\eta} U_{0}^{b}\right),
\end{aligned}
$$

with boundary conditions

$$
\left(\gamma_{0}^{b}\right)^{2} \partial_{\eta} U_{0}^{b}=\frac{f_{w}^{\prime}\left(U_{0}^{b}\right)}{\lambda_{m}}, \quad m\left(U_{0}^{b}\right) \partial_{\eta} M_{0}^{b}=0,
$$

at $\eta=0$. Considering $(76 \mathrm{a})$ we first observe that

$$
a_{1}(\tau, x)=m\left(U_{0}^{b}\right) \partial_{\eta} M_{0}^{b}
$$

where $a_{1}(\tau, x)$ is a constant of integration and including the no-flux boundary condition at $\eta=0$ it follows that $a_{1}(\tau, x)$ must be zero. This also implies that either $m\left(U_{0}^{b}\right)=0$ or $\partial_{\eta} M_{0}^{b}=0$. Considering $m\left(U_{0}^{b}\right) \neq 0$, which corresponds to the region about the interface, we obtain that $M_{0}^{b}$ is constant in $\eta$. Matching to $M_{0}=0$ away from the substrate, i.e. for $\eta \rightarrow \infty$, we conclude that $M_{0}^{b}$ must be zero as well. Note that $m\left(U_{0}^{b}\right)=0$ corresponds to the region where $U_{0}^{b}= \pm 1$, i.e. the outer region, where $M_{0}^{b}$ has to match with $M_{0}=0$ anyways. Consequently we obtain for (76b)

$$
0=F^{\prime}\left(U_{0}^{b}\right)-\partial_{\eta}\left(\left(\gamma_{0}^{b}\right)^{2} \partial_{\eta} U_{0}^{b}\right) .
$$

Multiplying by $\partial_{\eta} U_{0}^{b}$ and integrating over $\eta$ then yields

$$
\int F^{\prime}\left(U_{0}^{b}\right) \partial_{\eta} U_{0}^{b} d \eta=\int \partial_{\eta}\left(\left(\gamma_{0}^{b}\right)^{2} \partial_{\eta} U_{0}^{b}\right) \partial_{\eta} U_{0}^{b} d \eta
$$

which, since $\gamma_{0}^{b}$ is constant, leads to

$$
F\left(U_{0}^{b}\right)=\frac{1}{2}\left(\gamma_{0}^{b}\right)^{2}\left(\partial_{\eta} U_{0}^{b}\right)^{2}+a_{2}(\tau, x)
$$

As $U_{0}^{b}$ has to match to $u_{0} \equiv-1$ for $x \rightarrow-\infty, a_{2}$ must be zero as well, and consequently we have

$$
F\left(U_{0}^{b}\right)=\frac{1}{2}\left(\gamma_{0}^{b}\right)^{2}\left(\partial_{\eta} U_{0}^{b}\right)^{2}
$$

Contact line region Next we introduce an interior layer centered at the contact point $\left(x_{c}, 0\right)$. We choose inner coordinates which are stretched in both directions, i.e.

$$
\xi=\frac{x-x_{c}}{\epsilon}, \quad \eta=\frac{y}{\epsilon}
$$


and also the corresponding dependent variables and their expansions

$$
\begin{gathered}
U^{c}=U_{0}^{c}+\epsilon U_{1}^{c}+\epsilon^{2} U_{2}^{c} \cdots, \\
M^{c}=M_{0}^{c}+\epsilon M_{1}^{c}+\epsilon^{2} M_{2}^{c} \cdots
\end{gathered}
$$

Similar as before we have for $\gamma^{c}$ the expansion

$$
\begin{aligned}
& \gamma^{c}=\gamma\left(\theta_{0}^{c}\right) \quad+\epsilon \gamma^{\prime}\left(\theta_{0}^{c}\right) \theta_{1}^{c}+\ldots \\
& =: \gamma_{0}^{c}+\epsilon \gamma_{1}^{c}+\ldots
\end{aligned}
$$

where now we have

$$
\theta_{0}^{c}=\operatorname{atan} 2\left(\partial_{\eta} U_{0}^{c}, \partial_{\xi} U_{0}^{c}\right) .
$$

The leading order problem of $(12)$ then reads (with $\nabla^{\prime} \equiv\left(\partial_{\xi}, \partial_{\eta}\right)$ )

$$
\begin{aligned}
0 & =\nabla^{\prime}\left(m\left(U_{0}^{c}\right) \nabla^{\prime} M_{0}^{c}\right), \\
M_{0}^{c} & =F^{\prime}\left(U_{0}^{c}\right)-\nabla^{\prime}\left(\gamma_{0}^{c} \gamma_{0}^{c \prime}\left(\begin{array}{c}
-\partial_{\eta} U_{0}^{c} \\
\partial_{\xi} U_{0}^{c}
\end{array}\right)+\left(\gamma_{0}^{c}\right)^{2} \nabla^{\prime} U_{0}^{c}\right) .
\end{aligned}
$$

and we have the leading order boundary conditions

$$
\gamma_{0}^{c} \gamma_{0}^{c \prime} \partial_{\xi} U_{0}^{c}+\left(\gamma_{0}^{c}\right)^{2} \partial_{\eta} U_{0}^{c}=\frac{f_{w}^{\prime}\left(U_{0}^{c}\right)}{\lambda_{m}}, \quad m\left(U_{0}^{c}\right) \partial_{\eta} M_{0}^{c}=0
$$

at $\eta=0$. Considering an arbitrary rectangular box $\Omega_{R}$ in $\left[-R_{1} / 2, R_{1} / 2\right] \times\left[0, R_{2}\right]$ and integrating (85a) over this box we obtain from Gauss' theorem

$$
0=\int_{\Omega_{R}} \nabla^{\prime}\left(m\left(U_{0}^{c}\right) \nabla^{\prime} M_{0}^{c}\right) d \Omega=\int_{\partial \Omega_{R}}\left(m\left(U_{0}^{c}\right) \nabla^{\prime} M_{0}^{c}\right) \cdot \mathbf{n}_{\Omega_{R}} d \Gamma,
$$

which implies that $m\left(U_{0}^{c}\right) \partial_{\xi} M_{0}^{c}$ and $m\left(U_{0}^{c}\right) \partial_{\eta} M_{0}^{c}$ must be zero in the whole of $\left[-R_{1} / 2, R_{1} / 2\right] \times$ $\left[0, R_{2}\right]$. Considering $m\left(U_{0}^{c}\right) \neq 0$ this reveals that $M_{0}^{c}$ must be constant and matching to $M_{0}^{b}=0$ for $\xi \rightarrow \infty$ and to $M_{0}=0$ for $\eta \rightarrow \infty$ we obtain that $M_{0}^{c}$ must be zero as well. Conversely $m\left(U_{0}^{c}\right)=0$ corresponds to the pure phases where $M_{0}^{c}$ is constant as well and the same matching arguments lead to $M_{0}^{c}=0$. Consider now a box $R$ of size $R_{1}$ in the $\xi$-direction and $R_{2}$ in the $\eta$ direction (see Fig. 2). Multiplying (85b) by $\partial_{\xi} U_{0}^{c}$ and integrating over $R$ then leads to

$$
\begin{array}{r}
\iint_{R} \partial_{\xi} U_{0}^{c} F^{\prime}\left(U_{0}^{c}\right)=\iint_{R} \partial_{\xi} U_{0}^{c}\left[\partial_{\xi}\left(-\gamma_{0}^{c} \gamma_{0}^{c \prime} \partial_{\eta} U_{0}^{c}+\left(\gamma_{0}^{c}\right)^{2} \partial_{\xi} U_{0}^{c}\right)\right. \\
\left.+\partial_{\eta}\left(\gamma_{0}^{c} \gamma_{0}^{c \prime} \partial_{\xi} U_{0}^{c}+\left(\gamma_{0}^{c}\right)^{2} \partial_{\eta} U_{0}^{c}\right)\right]
\end{array}
$$

which can be rewritten as

$$
\begin{gathered}
(\mathrm{LHS}):=\iint_{R} \partial_{\xi}\left[F\left(U_{0}^{c}\right)+\frac{1}{2}\left(\gamma_{0}^{c}\right)^{2}\left(\partial_{\eta} U_{0}^{c}\right)^{2}-\frac{1}{2}\left(\gamma_{0}^{c}\right)^{2}\left(\partial_{\xi} U_{0}^{c}\right)^{2}+\gamma_{0}^{c} \gamma_{0}^{c \prime} \partial_{\xi} U_{0}^{c} \partial_{\eta} U_{0}^{c}\right] \\
=\iint_{R} \partial_{\eta}\left[\partial_{\xi} U_{0}^{c}\left(\gamma_{0}^{c} \gamma_{0}^{c \prime} \partial_{\xi} U_{0}^{c}+\left(\gamma_{0}^{c}\right)^{2} \partial_{\eta} U_{0}^{c}\right)\right]=:(\mathrm{RHS})
\end{gathered}
$$

where we exploited that

$$
\frac{1}{2} \partial_{\xi}\left(\gamma_{0}^{c}\right)^{2}=\gamma_{0}^{c} \gamma_{0}^{c \prime} \frac{\partial_{\xi} \partial_{\eta} U_{0}^{c} \partial_{\xi} U_{0}^{c}-\partial_{\xi \xi} U_{0}^{c} \partial_{\eta} U_{0}^{c}}{\left(\partial_{\xi} U_{0}^{c}\right)^{2}+\left(\partial_{\eta} U_{0}^{c}\right)^{2}}
$$


We first consider the left-hand side (LHS) of (88), integrate in $\xi$ and apply that $\partial_{\xi} U_{0}^{c} \rightarrow 0$ as $|\xi| \rightarrow \infty$, since $\int|\nabla u|^{2}$ is finite for finite energy solutions, giving

$$
\lim _{R_{1}, R_{2} \rightarrow \infty}(\mathrm{LHS})=\lim _{R_{1} \rightarrow \infty} \int_{0}^{\infty}\left[F\left(U_{0}^{c}\right)+\frac{1}{2}\left(\gamma_{0}^{c}\right)^{2}\left(\partial_{\eta} U_{0}^{c}\right)^{2}\right]_{-\frac{R_{1}}{2}}^{\frac{R_{1}}{2}} d \eta .
$$

In order to match $U_{0}^{c}$ with $U_{0}^{b}$ for large $\xi$ we have the matching conditions

$$
\begin{aligned}
& \lim _{\xi \rightarrow+\infty} U_{0}^{c}=\lim _{x \rightarrow x_{c}^{+}} U_{0}^{b}(x, \eta)=: U_{0}^{b+}\left(x_{c}, \eta\right), \\
& \lim _{\xi \rightarrow-\infty} U_{0}^{c}=\lim _{x \rightarrow x_{c}^{-}} U_{0}^{b}(x, \eta)=: U_{0}^{b-}\left(x_{c}, \eta\right),
\end{aligned}
$$

where $U_{0}^{b+}$ denotes the solution which corresponds to the side of the "+" phase and $U_{0}^{b-}$ the solution which corresponds to the side of the "-" phase. Moreover, recalling (80) and (75) we obtain

$$
\partial_{\eta} U_{0}^{b+}=\frac{1}{\left|\gamma_{0}^{b}\right|} \sqrt{2 F\left(U_{0}^{b+}\right)}, \quad \text { and } \quad \partial_{\eta} U_{0}^{b-}=-\frac{1}{\left|\gamma_{0}^{b}\right|} \sqrt{2 F\left(U_{0}^{b-}\right)}
$$

We then obtain for $(89)$

$$
\begin{aligned}
\lim _{R_{1}, R_{2} \rightarrow \infty}(\mathrm{LHS}) & =\int_{0}^{\infty} 2 F\left(U_{0}^{b+}\left(x_{c}, \eta\right)\right) d \eta-\int_{0}^{\infty} 2 F\left(U_{0}^{b-}\left(x_{c}, \eta\right)\right) d \eta \\
& =\sqrt{2}\left|\gamma_{0}^{b}\right|\left(\int_{0}^{1} \sqrt{F(t)} d t+\int_{0}^{-1} \sqrt{F(t)} d t\right) \\
& =\left|\gamma_{0}^{b}\right|\left(\frac{2}{3}-\frac{2}{3}\right)=0
\end{aligned}
$$

where we also applied the specific form of $F(u)=\frac{1}{2}\left(1-u^{2}\right)^{2}$.

Considering the right hand side (RHS) of (88) we first obtain after integrating in $\eta$ and including the boundary condition (85c)

$$
\begin{aligned}
(\mathrm{RHS}) & =\left[\int_{-R_{1} / 2}^{R_{1} / 2} \partial_{\xi} U_{0}^{c}\left(\gamma_{0}^{c} \gamma_{0}^{c \prime} \partial_{\xi} U_{0}^{c}+\left(\gamma_{0}^{c}\right)^{2} \partial_{\eta} U_{0}^{c}\right) d \xi\right]_{0}^{R_{2}} \\
& =\underbrace{\left.\int_{-R_{1} / 2}^{R_{1} / 2} \partial_{\xi} U_{0}^{c}\left(\gamma_{0}^{c} \gamma_{0}^{c \prime} \partial_{\xi} U_{0}^{c}+\left(\gamma_{0}^{c}\right)^{2} \partial_{\eta} U_{0}^{c}\right) d \xi\right|_{R_{2}}-\underbrace{\int_{-R_{1} / 2}^{R_{1} / 2} \partial_{\xi} U_{0}^{c} \frac{f_{w}^{\prime}\left(U_{0}^{c}\right)}{\lambda_{m}} d \xi}_{\text {II }}}_{\mathrm{I}}
\end{aligned}
$$

where (II) in the limit $R_{1}, R_{2} \rightarrow \infty$ is

$$
\lim _{R_{1}, R_{2} \rightarrow \infty}(\mathrm{II})=\frac{1}{\lambda_{m}} \int_{-1}^{1} f_{w}^{\prime}(t) d t=\frac{1}{\lambda_{m}}\left(\sigma_{F S}-\sigma_{V S}\right) .
$$

Analysing (I) we continue by transforming into a local coordinate system that is aligned with the tangent and normal direction to the film/vapor interface at $\left(x_{c}, 0\right)$ (see Fig. 3 ), that is

$$
\begin{array}{r}
\rho=-\xi \sin \alpha+\eta \cos \alpha \\
\varsigma=\xi \cos \alpha+\eta \sin \alpha
\end{array}
$$




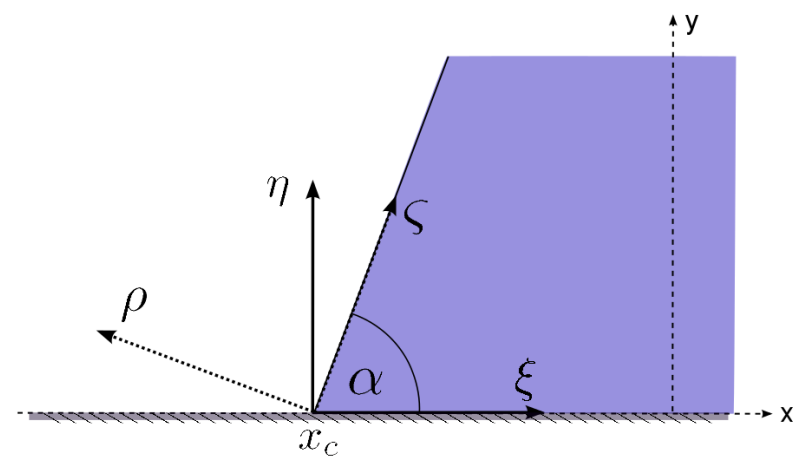

Figure 3: A sketch of the coordinate transformation.

and consequently

$$
\begin{aligned}
& \partial_{\xi}=-\sin \alpha \partial_{\rho}+\cos \alpha \partial_{\varsigma} \\
& \partial_{\eta}=\cos \alpha \partial_{\rho}+\sin \alpha \partial_{\varsigma} .
\end{aligned}
$$

Here $\alpha \in(0, \pi)$ denotes the contact angle on the right hand side of the thin solid film which has negative sign due to the geometric orientation (see Fig. 3). The transformed integral then reads

$$
(\mathrm{I})=\int_{\frac{R_{1}}{2} \sin \alpha+R_{2} \cos \alpha}^{-\frac{R_{1}}{2} \sin \alpha+R_{2} \cos \alpha} S d \rho
$$

where

$$
\begin{aligned}
S= & \gamma_{0}^{c} \gamma_{0}^{c \prime}\left(-\sin \alpha\left(\partial_{\rho} U_{0}^{c}\right)^{2}+2 \cos \alpha \partial_{\rho} U_{0}^{c} \partial_{\varsigma} U_{0}^{c}-\frac{\cos ^{2} \alpha}{\sin \alpha} \partial_{\varsigma}\left(U_{0}^{c}\right)^{2}\right) \\
& +\left(\gamma_{0}^{c}\right)^{2}\left(\cos \alpha \partial_{\rho}\left(U_{0}^{c}\right)^{2}+\left(\sin \alpha-\frac{\cos ^{2} \alpha}{\sin \alpha}\right) \partial_{\rho} U_{0}^{c} \partial_{\varsigma} U_{0}^{c}-\cos \alpha \partial_{\varsigma}\left(U_{0}^{c}\right)^{2}\right)
\end{aligned}
$$

For $\varsigma \rightarrow \infty$ the leading order $U_{0}^{c}$ has to match with the solution $U_{0}$ in (42). Since this is constant in $\varsigma$, we can conclude that $\lim _{\varsigma \rightarrow \infty} \partial_{\varsigma} U_{0}^{c}=0$. Taking the limit $R_{1} \rightarrow \infty, R_{2} \rightarrow \infty$ as in

$$
\lim _{R_{1}, R_{2} \rightarrow \infty}(\mathrm{I})=\lim _{\substack{a \rightarrow \infty \\ R_{1} \rightarrow \infty \\ R_{2} \rightarrow \infty}} \lim _{\left|R_{1} \sin \alpha+R_{2} \cos \alpha\right|<a} \quad \int_{\frac{R_{1}}{2} \sin \alpha+R_{2} \cos \alpha}^{-\frac{R_{1}}{2} \sin \alpha+R_{2} \cos \alpha} S d \rho,
$$

which ensures that we don't match "into the substrate", we obtain

$$
\lim _{R_{1}, R_{2} \rightarrow \infty}(\mathrm{I})=-\left(-\gamma_{0}^{c} \gamma_{0}^{c \prime} \sin \alpha+\left(\gamma_{0}^{c}\right)^{2} \cos \alpha\right) \int_{-\infty}^{\infty}\left(\partial_{\rho} U_{0}^{c}\right)^{2} d \rho
$$

where we applied that

$$
\begin{aligned}
& \lim _{\varsigma \rightarrow \infty} \gamma_{0}^{c}=\lim _{\varsigma \rightarrow \infty} \gamma\left(\theta_{0}^{c}\right)=\gamma(\operatorname{atan} 2(\cos \alpha,-\sin \alpha)), \\
& \lim _{\varsigma \rightarrow \infty} \gamma_{0}^{c^{\prime}}=\lim _{\varsigma \rightarrow \infty} \gamma^{\prime}\left(\theta_{0}^{c}\right)=\gamma^{\prime}(\operatorname{atan} 2(\cos \alpha,-\sin \alpha)),
\end{aligned}
$$


and consequently $\lim _{\varsigma \rightarrow \infty} \gamma_{0}^{c}$ and $\lim _{\varsigma \rightarrow \infty} \gamma_{0}^{c^{\prime}}$ are constant in $\varsigma$ and $\rho$. Moreover, note that $\theta_{0}^{c}$ and $\alpha$ are related by

$$
\theta_{0}^{c}=\left\{\begin{array}{ll}
\alpha+\frac{\pi}{2} & \text { for } \alpha \leq \frac{\pi}{2} \\
\alpha-\frac{3 \pi}{2} & \text { for } \alpha>\frac{\pi}{2}
\end{array} .\right.
$$

Recalling that $S$ corresponds to the integrand of (I) in (RHS) evaluated at $\eta=R_{2}$, we obtain that for large $R_{2}$ (due to matching to (80))

$$
\partial_{\rho} U_{0}^{c}=-\frac{1}{\gamma_{0}} \sqrt{2 F\left(U_{0}^{c}\right)}
$$

which reveals that

$$
\begin{aligned}
\int_{-\infty}^{\infty}\left(\partial_{\rho} U_{0}^{c}\right)^{2} d \rho & =-\frac{\sqrt{2}}{\gamma_{0}^{2}} \int_{-\infty}^{\infty} \sqrt{F\left(U_{0}^{c}\right)} \partial_{\rho} U_{0}^{c} d \rho \\
& =-\frac{\sqrt{2}}{\gamma_{0}} \int_{1}^{-1} \sqrt{F(t)} d t=\frac{1}{\gamma_{0}} \frac{4}{3}
\end{aligned}
$$

By merging the results for (LHS) and (RHS) in (88) we obtain

$$
0=\frac{4}{3}\left(-\gamma_{0}^{\prime} \sin \alpha+\gamma_{0} \cos \alpha\right)-\frac{1}{\lambda_{m}}\left(\sigma_{V S}-\sigma_{F S}\right)
$$

with $\alpha \in(0, \pi)$, which is, after applying the correct mixing energy $\lambda_{m}$, referred to as YoungHerring condition. Note that the same condition holds at the right contact point if we replace $\alpha$ by $\alpha_{R}$. Herring [22] derived the anisotropic contact angle condition for the interception point of up to three interfaces by the method of virtual displacement. Another derivation of (104) is given in [38] where a variational approach is applied with the addition of mass conservation.

If the surface energy is isotropic, i.e. $\gamma=1$, then (104) reduces to the Young's equation (6) if we notice that the film/vapor interface energy $\sigma_{F V}$ in this case is given by the integral of the square of the gradient of the inner solution across the interface layer, that is, by $\lambda_{m}$ times the integral in (103), see for example [39]; thus $\sigma_{F V}=4 \lambda_{m} / 3$. Moreover, in the case of weak anisotropy, $\gamma+\gamma^{\prime \prime}>0$, equation (104) has a unique solution $\alpha$, since then, the right hand side is a strictly monotonically decreasing function of $\alpha \in(0, \pi)$ as can be seen by taking the derivative with respect to $\alpha$.

Balance of flux condition For the sake of completeness we also need a balance of flux condition which matches the flux of the boundary layer near $\Gamma_{w}$ to the flux in the outer region. To this end let $\mathbf{J}^{b}$ be the flux in the boundary layer near $\Gamma_{w}$ and let $\mathbf{J}^{c}$ be the flux in the contact line region. Similar as before, consider now a box $R$ of size $R_{1}$ in the $\xi$-direction and $R_{2}$ in the $\eta$ direction (see Fig. 2). The size will later be taken to infinity while still ensuring that it remains within the inner region, that is, $\epsilon R_{1} \ll 1, \epsilon R_{2} \ll 1$. By the divergence theorem and the no-flux condition at the wall we have that

$$
\int_{0}^{R_{2}}\left[\mathbf{J}^{c} \cdot \mathbf{e}_{\xi}\right]_{-R_{1} / 2}^{R_{1} / 2} d \eta+\left.\int_{-R_{1} / 2}^{R_{1} / 2} \mathbf{J}^{c} \cdot \mathbf{e}_{\eta}\right|_{R_{2}} d \xi=0 .
$$

On the one hand, the terms in the expansion of $\left.\mathbf{J}^{c} \cdot \mathbf{e}_{\xi}\right|_{\infty}$ have to match with $\mathbf{J}^{b} \cdot \mathbf{e}_{x}$ away from the contact point $\left(x_{c}, 0\right)$, i.e.

$$
\mathbf{J}^{b} \cdot \mathbf{e}_{x}=m\left(U^{b}\right) \partial_{x} M^{b} .
$$


Since we may assume that for large $R_{1}$ we have $U_{0}^{b} \equiv \pm 1$, which implies $m\left(U_{0}^{b}\right)=0$ as well as $m^{\prime}\left(U_{0}^{b}\right)=0$, and recalling that $M_{0}^{b}=0$ we obtain that the expansions for $\mathbf{J}^{b}$ are zero up to at least $O\left(\epsilon^{3}\right)$. On the other hand, the terms in $\left.\mathbf{J}^{c} \cdot \mathbf{e}_{\eta}\right|_{\infty}$ have to match with those in $\left.\mathbf{J} \cdot \mathbf{e}_{y}\right|_{0}$. According to (17) we know that $\mathbf{J} \cdot \mathbf{e}_{y}$ in curvilinear coordinates reads

$$
\mathbf{J} \cdot \mathbf{e}_{y}=m(U)\left[n_{2} \epsilon^{-1} \partial_{\rho} M(U)-\frac{n_{1}}{1+\epsilon \rho \kappa} \partial_{s} M(U)\right],
$$

and since $M_{0} \equiv 0$ and $M_{1}, M_{2}$ are independent of $\rho$, the dominant terms of (107) are of $O\left(\epsilon^{2}\right)$

$$
\mathbf{J} \cdot \mathbf{e}_{y}=\epsilon^{2} m\left(U_{0}\right)\left(n_{2} \partial_{\rho} M_{3}-n_{1} \partial_{s} M_{1}\right)+O\left(\epsilon^{3}\right),
$$

In total, therefore, the leading order condition that follows from (105) is

$$
\begin{aligned}
0 & =\left.\int_{-\infty}^{\infty} m\left(U_{0}\right)\left(n_{2} \partial_{\rho} M_{3}-n_{1} \partial_{s} M_{1}\right)\right|_{R_{2}} d \rho \\
& =C \partial_{s}\left[\left(\gamma_{0}+\gamma_{0}^{\prime \prime}\right) \kappa\right] \int_{-\infty}^{\infty} m\left(U_{0}\right) d \rho
\end{aligned}
$$

where $C$ is a constant and we have used (69), (61) and also assumed to pass over in a similar way as in (96) in order to stay inside the box all the time. By virtue of (42), the integral is finite, thus

$$
\partial_{s}\left[\left(\gamma_{0}+\gamma_{0}^{\prime \prime}\right) \kappa\right]=0
$$

at the contact line. Notice that via (61), this condition is equivalent to requiring the leading order tangential flux (from surface diffusion) along the interface $\Gamma$ to be zero at the contact line.

\section{Numerical results}

In this section we present the numerical results from a finite element based simulation in MATLAB. The system is solved by an operator splitting ansatz and exploits a diffuse boundary approximation at the solid substrate, similar as in $[36,35]$. To be more precise, for the numerical consideration, we introduce an additional boundary layer at $\Gamma_{w}$ with thickness $\epsilon_{y} \ll 1$ and use a surface delta function for the wall energy density (4) such that we can formally rewrite the total free energy (2) on $\Omega_{1}$ in one integral

$$
W^{\epsilon}=\int_{\Omega_{1}} f_{F V}+\delta_{\Gamma_{w}} f_{w} d \Omega_{1}
$$

where $\delta_{\Gamma_{w}}$ satisfies

$$
\int_{\Omega_{1}} h \delta_{\Gamma_{w}} d \Omega_{1} \approx \int_{\Gamma_{w}} h d \Gamma
$$

for any smooth function $h$. Calculating the first variation of the free energy functional thus leads to the chemical potential

$$
\mu=F^{\prime}(u)+\delta_{\Gamma_{w}} \epsilon \frac{f_{w}^{\prime}}{\lambda_{m}}-\epsilon^{2} \nabla \cdot\left(\gamma \gamma^{\prime}\left(\begin{array}{c}
-u_{y} \\
u_{x}
\end{array}\right)+\gamma^{2} \nabla u\right),
$$

which entails that the natural boundary conditions for the approximate problem are simple homogeneous Neumann boundary conditions

$$
\begin{aligned}
& \frac{\partial u}{\partial y}=0 \\
& \frac{\partial \mu}{\partial y}=0
\end{aligned}
$$


a)

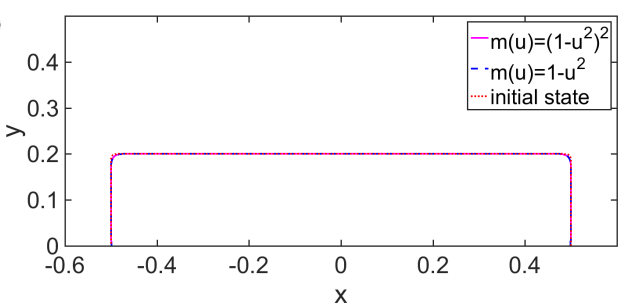

c)

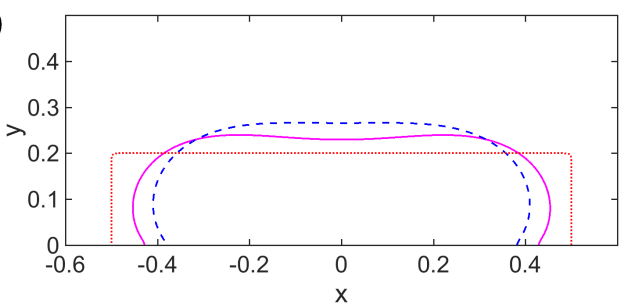

e)

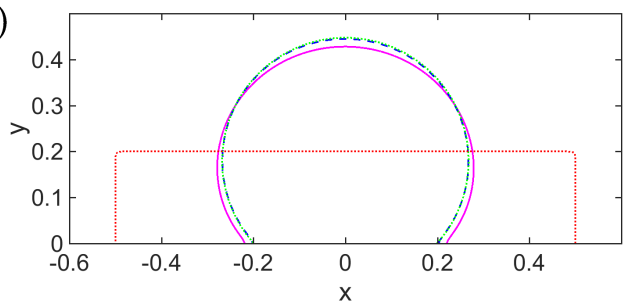

b)

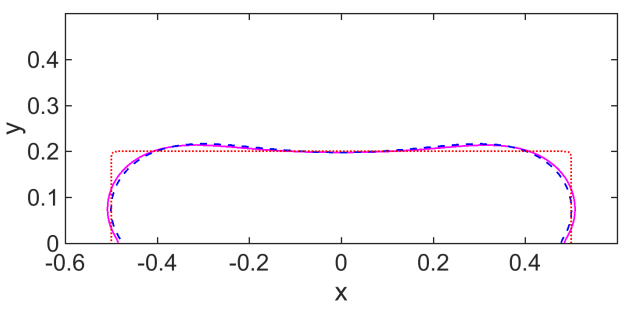

d)

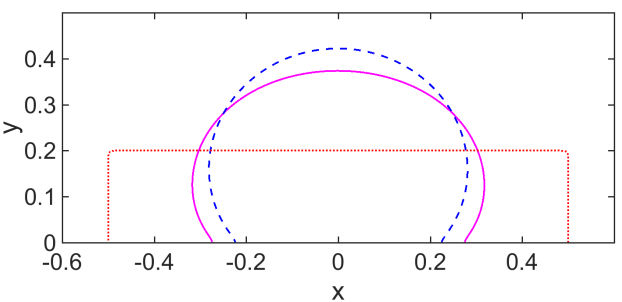

f)

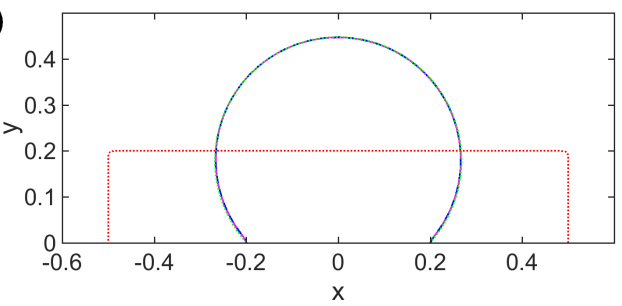

Figure 4: Numerical result showing the evolution of two thin films with different mobility where $\epsilon=0.01$ at a) $t=0$, b) $t=1$, c) $t=10$, d) $t=50$, e) $t=100, \mathrm{f}) t=150$. In c) it is clearly visible how the film corresponding to the mobility $m(u)=\left(1-u^{2}\right)^{2}$ (pink solid line) forms a little valley in the middle whereas the film corresponding to the mobility $m(u)=1-u^{2}$ (blue dashed line) moves upwards at $x=0$. In e) and $\mathrm{f}$ ) the corresponding equilibrium shape, which is a truncated circle, is displayed (green dotted line), which shows, that the film corresponding to the mobility $m(u)=1-u^{2}$ achieves its equilibrium much faster.

at $y=0$. Note that these are easy to handle from a numerical point of view. It remains to show that the present model recovers the original equation (11) subject to the boundary conditions (11e) and (11f), which can be done via matched asymptotic expansions but but which we don't want to discuss further at this point.

As a discrete basis we implement standard piecewise linear finite elements (P1). We choose the grid width according to the size of $\epsilon$. In particular, in order to achieve a good resolution we choose $d x=d y \approx \epsilon / 5$. Moreover we discretize the time via

$$
\partial_{t} u \approx \frac{u(\mathbf{x}, t+\tau)-u(\mathbf{x}, t)}{\tau}
$$

where $\tau$ is the time-step size and treat most of the nonlinear functions, such as $m(u) \nabla \mu$ and the anisotropic part of $\mu$, semi-implicitly in time. The only nonlinear functions which are treated explicitly in time are $F^{\prime}(u)$ and the part corresponding to the diffuse boundary approximation in $\mu$. The resulting code turned out to have good stability properties for time-step sizes $\tau=O\left(\epsilon^{2}\right)$. 
The main aim of the present Section is to show how the mobility influences the evolution. As motivated in the introduction, the frequently applied mobility $m(u)=1-u^{2}$ does not lead to motion by pure surface diffusion in the sharp interface limit of the present model. Instead, the sharp interface limits which we derived in Section 3 show that the mobility $(11 \mathrm{~d})$, i.e. $m(u)=$ $\left(1-u^{2}\right)^{2}$ turns out to recover this physical process correctly. In the following we will document the difference of these two particular mobilities by comparing the zero-level sets of the corresponding phase field functions.

We start with a comparison motivated by a simulation given in [25]. Considering the isotropic case of (11a)- (11f), Jiang et. al simulate the evolution of a retracting solid film with mobility $m(u)=1-u^{2}$, as shown in Fig. 4 in [25]. In order to compare to this result, we chose a similar setting, i.e. we confine ourselves to the isotropic case, define an initial state which is a rectangle, located in $[-0.5,0.5] \times[0,2]$ of a $[-0.7,0.7] \times[0,0.7]$ computational domain, chose $\epsilon=0.01$ and the Young contact angle $\alpha=3 \pi / 4$. The mesh size is $d x=d y=0.002$ and the time step is fixed as $\tau=5 \cdot 10^{-5}$. The equilibrium shape of the thin film island is again a predictable truncated circle.

The simulation, see Fig. 4, shows how the film which corresponds to the mobility $m(u)=$ $1-u^{2}$ evolves clearly faster than the film corresponding to $m(u)=\left(1-u^{2}\right)^{2}$. In particular the film with $m(u)=1-u^{2}$ achieves its equilibrium at approximately $t=100$, see Fig. $4 \mathrm{e}$ ), whereas the film corresponding to $m(u)=\left(1-u^{2}\right)^{2}$ needs significantly longer. Furthermore the evolution corresponding to $m(u)=1-u^{2}$ is in good qualitative agreement with the simulation given in Fig. 4 in the reference [25], which suggests the correctness of the numerical algorithm.

Observing that the evolution corresponding to the model with $m(u)=\left(1-u^{2}\right)^{2}$ is not only slower in general but also differs qualitatively in view of the valley which forms in the middle of the film, see Fig. $4 \mathrm{c}$ ), the question naturally arises as to whether there is a critical film length at which the faster film contracts to a single droplet whereas the slower film pinches off. The answer is yes, as shown in Figure 5. Here we simulated a fourfold anisotropic surface energy with strength $G=0.05$. The other parameters are $\theta_{c}=3 \pi / 4, \epsilon=0.02, d x=d y=0.002$ and $\tau=0.001$. As expected the film corresponding to $m(u)=1-u^{2}$ forms a single equilibrium crystal, whereas the film corresponding to $m(u)=\left(1-u^{2}\right)^{2}$ pinches off. The Figure shows in addition the exact equilibrium shape, which is determined by the Winterbottom construction $[59]$.

\section{Conclusion}

In the present work we have investigated a two-dimensional phase-field model describing the dewetting of a solid film from a solid substrate. The main goal was to establish the connection between the phase-field model and the corresponding sharp-interface model in the limit as $\epsilon \rightarrow 0$ for a mobility where surface diffusion is recovered as the dominant driving mechanism for the contact-line dynamics of the dewetting film. This requires an asymptotic analysis that allows us to incorporate multiple boundary and interfacial layers that occur in the sharp-interface limit as well as techniques of exponential matching, both in the isotropic and the anisotropic case.

We established that by using exponential asymptotic matching the bi-quadratic mobility combined with the bi-quadratic bulk free energy density yields the correct limiting model as $\epsilon \rightarrow 0$. We note that this is in contrast to the frequently applied quadratic mobility, which leads to sharp-interface models, where a contribution from a non-linear, porous medium like bulk diffusion enters the driving force at the same order of magnitude as surface diffusion, as it also has previously been shown in [34]. 
a)

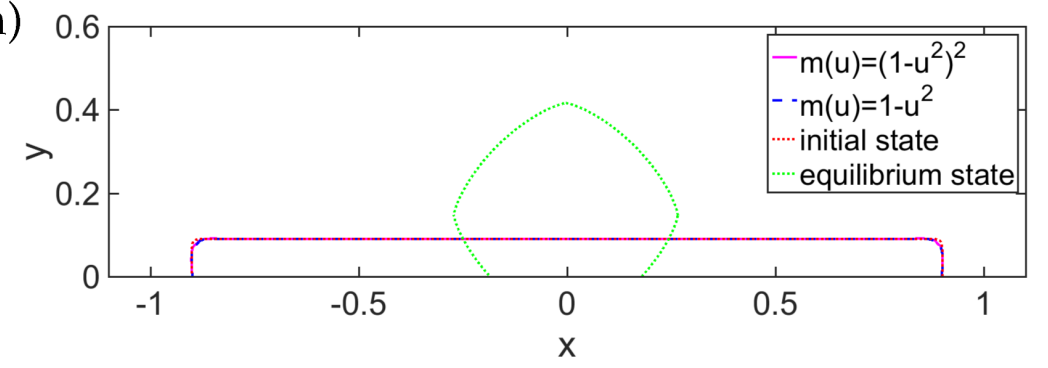

b)

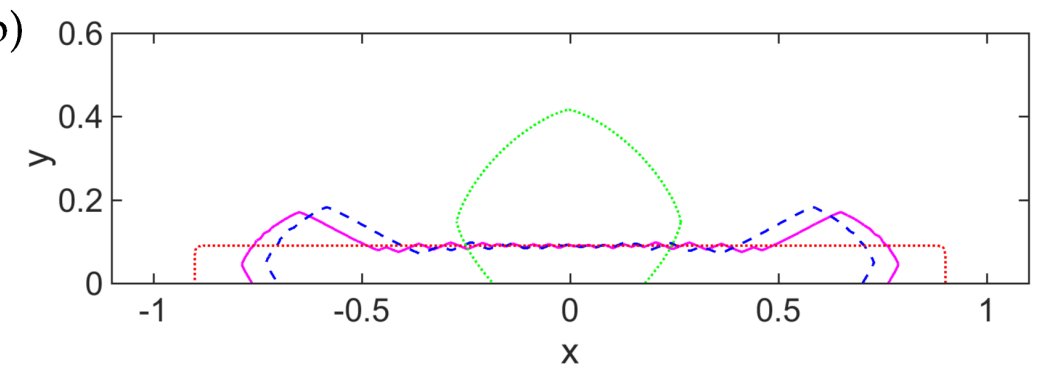

c)

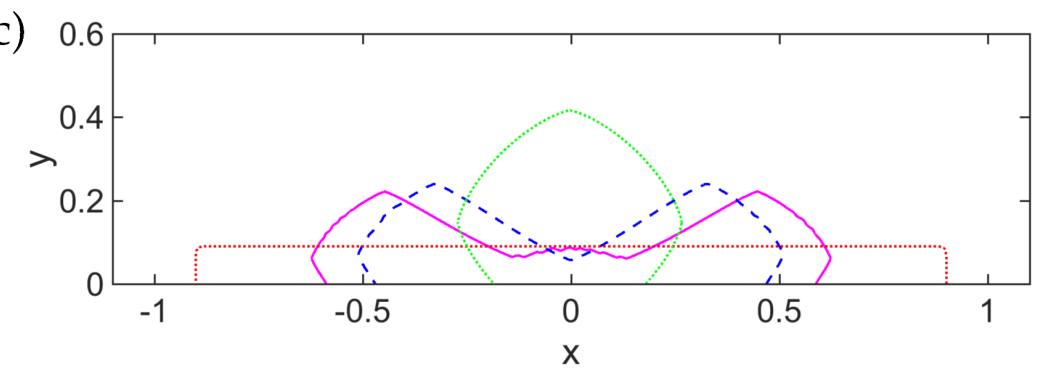

d)

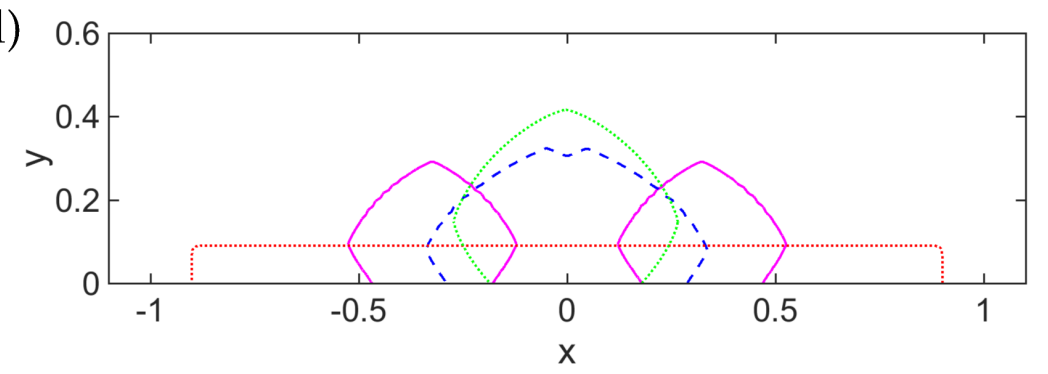

e)

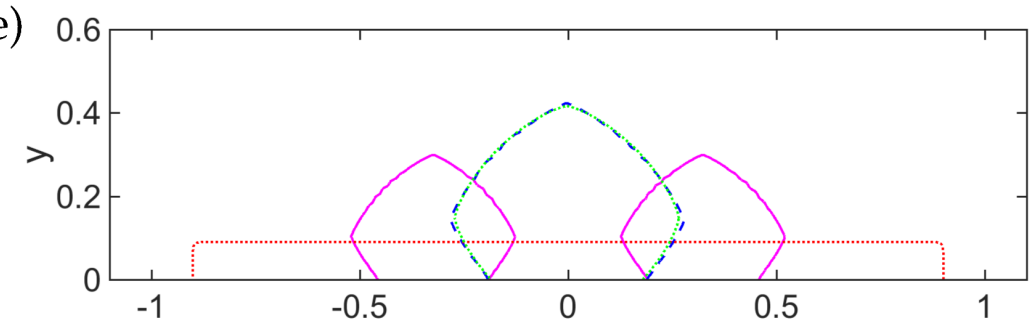

Figure 5: Numerical comparison between the evolution with mobility $m(u)=\left(1-u^{2}\right)^{2}$ and $m(u)=1-u^{2}$ where $\epsilon=0.02, d x=d y=0.002$ and $\tau=0.001$ at a) $t=0$, b) $t=1$, c) $\left.t=5, \mathrm{~d}\right)$ $t=10$ and e) $t=20$. The mobility $m(u)=1-u^{2}$ leads to the formation of one single crystal whereas the mobility $m(u)=\left(1-u^{2}\right)^{2}$ results in film pinch-off. 
In addition, since the solid dewetting problem considered here includes boundary conditions at a solid substrate, another appropriate matching procedure has to be provided in order to derive the sharp interface limits at this solid boundary. We introduced another inner layer about the boundary $\Gamma_{w}$ and presented an asymptotic analysis which refers to a particular geometry allowing to match the inner and outer layers without matching "into the substrate", which is not defined. The result is that the sharp interface limits of the boundary conditions at the substrate recover the Young-Herring equation for the contact angle, and Young's equation in the isotropic case.

Finally, we presented numerical simulations for various initial states which address the question of how the mobility influences the evolution. We compared the results with mobility (11d) to the simulations with mobility $m(u)=1-u^{2}$ and demonstrated a significant difference. Considering a fourfold symmetry, for example, leads to film pinch-off in the one case and complete film retraction in the other case.

The work here has focused on the two-dimensional phase-field formulation, while practical situations are three dimensional. Generalisation of the phase-field model to three dimensions require expressing the surface tension coefficient $\gamma$ dependence directly in terms of $\nabla u$ instead of through $\theta$. We anticipate that the sharp interface limit will lead to 3D generalisations of $(70)$, see [5] and [35] and references therein.

We also note that from liquid dewetting studies it is known that the, typically degenerate, mobility of the governing fourth order parabolic thin film equation does not only control the dewetting rates but also decides the morphology and scale of the contact-line instability that arises eventually [2]. In principle, similar scenarios have to be explored here. Moreover, in combination with the anisotropic nature of the solid film, such as for example $\mathrm{Si}$, the evolution of the contact line instability becomes particularly interesting and, according to experimental results [15], depends on the crystalline orientation relative to the contact line. For comparisons to realistic experimental results of dewetting solid films, such as crystalline Si films used for nanopatterning surfaces, the extension of the present phase field model to three space dimensions is desirable.

\subsection{Acknowledgements}

The authors are very grateful to Dirk Peschka for fruitful discussions on the numerical part of our study. MD gratefully acknowledges the support by the Federal Ministry of Education (BMBF) and the state government of Berlin (SENBWF) in the framework of the program Spitzenforschung und Innovation in den Neuen Ländern (Grant Number 03IS2151).

\section{A Inner expansions for $\theta$}

We first consider $u_{x}=0$. In inner coordinates this is equivalent to

$$
\epsilon^{-1} n_{1} U_{\rho}+(1+\epsilon \rho \kappa)^{-1} t_{1} U_{s}=0
$$

for all $\epsilon>0$, which can be rewritten to

$$
n_{1} U_{\rho}+\epsilon\left(\rho \kappa n_{1} U_{\rho}+t_{1} U_{s}\right)=0 .
$$

As this is a polynomial in $\epsilon$ it is zero for all $\epsilon$ if an only if

$$
n_{1} U_{\rho}=0 \wedge \rho \kappa n_{1} U_{\rho}+t_{1} U_{s}=0 .
$$


Since we consider the inner problem at the interface, which describes phase transition we can assume that $U_{\rho} \neq 0$ and the condition can be rewritten as

$$
n_{1}=0 \wedge U_{s}=0,
$$

where we also exploited that $t_{1}=n_{2} \neq 0$ since $\left(n_{1}, n_{2}\right)=0$ cannot occur in inner coordinates, i.e. near the interface. Consequently we obtain that from $u_{x}=0$ it follows that $n_{1}=-t_{2}=0$ in inner coordinates, thus $u_{y}$ reads

$$
u_{y}=\epsilon^{-1} n_{2} U_{\rho}+(1+\epsilon \rho \kappa)^{-1} t_{2} U_{s}=\epsilon^{-1} n_{2} U_{\rho} .
$$

Exploiting the definition of $\theta$, i.e. (9) we then obtain that for $u_{x}=0$ we have

$$
\theta=\theta_{0}\left(u_{y}\right)= \begin{cases}+\frac{\pi}{2} & \text { for } n_{2} U_{\rho}>0 \\ -\frac{\pi}{2} & \text { for } n_{2} U_{\rho}<0\end{cases}
$$

where we also exploited that $n_{2} U_{\rho} \neq 0$. Finally, since $\gamma(\theta)=\gamma(-\theta)$ we obtain that $\gamma=\gamma_{0}=\gamma\left(\theta_{0}\right)$ is constant and in particular independent of $\rho$.

We now consider $u_{x} \neq 0$.

According to $(110)$ this implies either $n_{1} \neq 0$ or $U_{s} \neq 0$. We first consider $n_{1} \neq 0$. In inner coordinates and exploiting $\left(t_{1}, t_{2}\right)=\left(n_{2},-n_{1}\right)$, as well as $n_{1}^{2}+n_{2}^{2}=1$, we have

$$
\begin{aligned}
\frac{u_{y}}{u_{x}} & =\frac{\epsilon^{-1} n_{2} U_{\rho}+(1+\epsilon \rho \kappa)^{-1} t_{2} U_{s}}{\epsilon^{-1} n_{1} U_{\rho}+(1+\epsilon \rho \kappa)^{-1} t_{1} U_{s}}=\frac{\epsilon^{-1} n_{2} U_{\rho}+\rho \kappa n_{2} U_{\rho}+t_{2} U_{s}}{\epsilon^{-1} n_{1} U_{\rho}+\rho \kappa n_{1} U_{\rho}+t_{1} U_{s}} \\
& =\frac{n_{2} U_{\rho}+\epsilon\left(\rho \kappa n_{2} U_{\rho}+t_{2} U_{s}\right)}{n_{1} U_{\rho}+\epsilon\left(\rho \kappa n_{1} U_{\rho}+t_{1} U_{s}\right)} \sim \frac{n_{2}}{n_{1}}-\epsilon \frac{U_{s}}{n_{1}^{2} U_{\rho}} .
\end{aligned}
$$

A Taylor-expansion of $\theta$ at $\epsilon=0$ then leads to

$$
\theta=\operatorname{atan} 2\left(n_{2}, n_{1}\right)-\epsilon \frac{U_{s}}{U_{\rho}}+O\left(\epsilon^{2}\right)
$$

which reveals the identification

$$
\theta_{0}=\operatorname{atan} 2\left(n_{2}, n_{1}\right) \quad \text { and } \quad \theta_{1}=-\frac{U_{s}}{U_{\rho}} .
$$

On the other hand, for $n_{1}=-t_{2}=0$ and $U_{s} \neq 0$, we have

$$
\frac{u_{y}}{u_{x}}=\epsilon^{-1} \frac{U_{\rho}}{U_{s}}+\rho \kappa \frac{U_{\rho}}{U_{s}}
$$

such that in the limit $\epsilon \rightarrow 0$ we obtain

$$
\theta=\theta_{0}=\operatorname{sign}\left(\frac{U_{\rho}}{U_{s}}\right) \frac{\pi}{2} .
$$

Finally we conclude that

$$
\theta_{0}= \begin{cases}\operatorname{atan} 2\left(n_{2}, n_{1}\right) & \text { for } n_{1} \neq 0 \\ \pm \frac{\pi}{2} & \text { for } n_{1}=0\end{cases}
$$

and

$$
\theta_{1}=\left\{\begin{aligned}
-\frac{U_{s}}{U_{\rho}} & \text { for } n_{1} \neq 0 \\
0 & \text { for } n_{1}=0
\end{aligned}\right.
$$




\section{B Inner expansion for $\theta_{1}$ in view of $U_{0}$}

We consider $n_{1} \neq 0$. From (25) we already know that

$$
\theta_{1}=-\frac{U_{s}}{U_{\rho}} \sim-\frac{\partial_{s} U_{0}}{\partial_{\rho} U_{0}}
$$

Exploiting the leading order representation of $U$, i.e.

$$
U_{0}=-\tanh \left(\frac{1}{\gamma_{0}} \rho\right)
$$

we calculate

$$
\begin{aligned}
& \partial_{s} U_{0}=\left(1+U_{0}^{2}\right) \frac{\rho \partial_{s} \gamma_{0}}{\gamma_{0}^{2}} \\
& \partial_{\rho} U_{0}=-\left(1+U_{0}^{2}\right) \frac{1}{\gamma_{0}}
\end{aligned}
$$

and since $\gamma_{0}=\gamma\left(\operatorname{atan} 2\left(n_{2}, n_{1}\right)\right)$ we obtain from the Frenet-Serret formulae $(16)$

$$
\partial_{s} \gamma_{0}=\gamma_{0}^{\prime} \frac{n_{1} \partial_{s} n_{2}-n_{2} \partial_{s} n_{1}}{n_{1}^{2}+n_{2}^{2}}=-\gamma_{0}^{\prime} \kappa
$$

Applying (118) and (119) in (117) then gives

$$
\theta_{1} \sim-\frac{\gamma_{0}^{\prime}}{\gamma_{0}} \rho \kappa
$$

Note that in the case $n_{1}=0$ the leading order of $\gamma$ is constant and in particular independent of $s$ and $\rho$. Consequently the representation (120) can be applied to this case as well as it is zero and this is consistent with (116).

\section{References}

[1] N. D. Alikakos, P. W. Bates, and X. Chen. Convergence of the Cahn-Hilliard equation to the Hele-Shaw model. Archive for Rational Mechanics and Analysis, 128(2):165-205, 1994.

[2] O. Bäumchen, L. Marquant, R. Blossey, A. Münch, B. Wagner, and K. Jacobs. Influence of slip on the Rayleigh-Plateau rim instability in dewetting viscous films. Physical Review Letters, 113:014501, 2014.

[3] S. Bottin-Rousseau, M. Serefoglu, S. Akamatsu, and G. Faivre. The surface tension force of anisotropic interphase boundaries is perpendicular to the solidification front during eutectic growth. IOP Conf. Series: Materials Science and Engineering, 27:012088, 2011.

[4] L. Bronsard and F. Reitich. On three-phase boundary motion and the singular limit of a vector-valued Ginzburg-Landau equation. Arch. Rat. Mech. Anal., 124:355-379, 1993.

[5] J. W. Cahn and D. W. Hoffman. A vector thermodynamics for anisotropic surfaces ii. curved and faceted surfaces. Acta Metall. Mater., 22:1205-1214, 1974.

[6] J. W. Cahn and J. E. Taylor. Surface motion by surface diffusion. Acta Metall. Mater., 42(4):1045-1063, 1994. 
[7] J.W. Cahn, C.M. Elliott, and A. Novick-Cohen. The Cahn-Hilliard equation with a concentration dependent mobility: motion by minus the Laplacian of the mean curvature. Europ. Jour. Appl. Math., 7:287-302, 1996.

[8] R. V. Craster and O. K. Matar. Dynamics and stability of thin liquid films. Rev. Mod. Phys., 81:1131-1198, 2009.

[9] S. Dai and Q. Du. Motion of Interfaces Governed by the Cahn-Hilliard Equation with Highly Disparate Diffusion Mobility. SIAM Journal on Applied Mathematics, 72(6):1818$1841,2012$.

[10] S. Dai and Q. Du. Coarsening Mechanism for Systems Governed by the Cahn-Hilliard Equation with Degenerate Diffusion Mobility. Multiscale Modeling 8 Simulation, 12(4):1870$1889,2014$.

[11] Shibin Dai and Qiang Du. Weak Solutions for the Cahn-Hilliard Equation with Degenerate Mobility. Archive for Rational Mechanics and Analysis, 219(3):1161-1184, 2015.

[12] Shibin Dai and Qiang Du. Computational studies of coarsening rates for the Cahn-Hilliard equation with phase-dependent diffusion mobility. Journal of Computational Physics, 310:85-108, 2016.

[13] D. T. Danielson, D. K. Sparacin, J. Michel, and L. C. Kimerling. Surface-energydriven dewetting theory of silicon-on-insulator agglomeration. Journal of applied physics, 100:083507, 2006.

[14] E. Dornel, J-C. Bare, F. de Crecy, and G. Lacolle. Surface diffusion dewetting of thin solid films: Numerical method and application to Si/SiO 2 . Phys. Rev. B, 73:115427, 2006.

[15] M. Dufay and O. Pierre-Louis. Anisotropy and coarsening in the instability of solid dewetting fronts. Phys. Rev. Lett., 106(10):105506, 2011.

[16] J.J. Eggleston, G.B. McFadden, and P. W. Voorhees. A phase-field model for highly anisotropic interfacial energy. Phys. D, 150:91-103, 2001.

[17] C. M. Elliott and H. Garcke. On the Cahn-Hilliard Equation with Degenerate Mobility. SIAM Journal on Mathematical Analysis, 27(2):404-423, 1996.

[18] H. Garcke, B. Nestler, and B. Stoth. On anisotropic order parameter models for multi-phase systems and their sharp interface limits. Physica D: Nonlinear Phenomena, 115(1-2):87-108, 1998.

[19] H. Garcke and A. Novick-Cohen. A singular limit for a system of degenerate Cahn-Hilliard equations. Adv. Differential Equations, 5:401-434, 2000.

[20] C. Guggenberger, R. Spatschek, and K. Kassner. Comparison of phase-field models for surface diffusion. Phys. Rev. E, 78:016703, 2008.

[21] M. G. Hennessy, V. M. Burlakov, A. Goriely, B. Wagner, and A. Münch. Controlled topological transitions in thin film phase separation. WIAS preprint 1885, 2013.

[22] C. Herring. Surface tension as a motivation for sintering. In W. E. Kingston, editor, The physics of Powder Metallurgy, A Symposium held at Bayside, L.I. New York, 24-26 August, 1949, pages 143-179. McGraw-Hill, New York, 1951. 
[23] D. Jacqmin. Calculation of two-phase Navier-Stokes flows using phase-field modelling. $J$. Comput. Phys., 155:96-127, 1999.

[24] D. Jacqmin. Contact-line dynamics of a diffuse fluid interface. J. Fluid Mech., 402:57-88, 2000.

[25] W. Jiang, W. Bao, C. V. Thompson, and D. J. Srolovitz. Phase field approach for simulating solid-state dewetting problems. Acta Mater., 60:5578- 5592, 2012.

[26] E. Jiran and C. V. Thompson. Capillary instabilities in thin films. Journal of Electronic Materials, 19(11):1153-1160, 1990.

[27] M. Khenner, R.J. Braun, and M.G. Mauk. A model for anisotropic epitaxial lateral overgrowth. J. Cryst. Gr., 241:330-346, 2002.

[28] J. R. King, A. Münch, and B. Wagner. Linear stability analysis of a sharp-interface model for dewetting thin films. J. Engrg. Math., 63:177-195, 2009.

[29] R. Kobayashi. Modeling and numerical simulations of dendritic crystal growth. Physica D, 63:410-423, 1993.

[30] M. D. Korzec, P.L. Evans, A. Münch, and B. Wagner. Stationary solutions of driven fourthand sixth-order cahn-hilliard-type equations. SIAM J. Appl. Math., 69:348-374, 2008.

[31] M. D. Korzec, M Roczen, M. Schade, B. Wagner, and B. Rech. Equilibrium shapes of polycrystalline silicon nanodots. J. Appl. Phys., 115:(074304) 1-13, 2014.

[32] C. G. Lange. On spurious solutions of singular perturbation problems. Studies Appl. Math., 68:227-257, 1983.

[33] A. A. Lee, A. Münch, and E. Süli. Degenerate mobilities in phase field models are insufficient to capture surface diffusion. Applied Physics Letters, 107(8):081603, 2015.

[34] A. A. Lee, A. Münch, and E. Süli. Sharp-Interface Limits of the Cahn-Hilliard Equation with Degenerate Mobility. SIAM Journal on Applied Mathematics, 76(2):433-456, 2016.

[35] X. Li, J. Lowengrub, A Rätz, and A. Voigt. Geometric evolution laws for thin crystalline films: Modeling and numerics. Commun. Comp. Phys., 6:433-482, 2009.

[36] X. Li, J. Lowengrub, A Rätz, and A. Voigt. Solving pdes in complex geometries: a diffuse domain approach. Commun. Math. Sci., 7:81-107, 2009.

[37] G. B. McFadden, A. A. Wheeler, R. J. Braun, S. R. Coriell, and R. F. Sekerka. Phase-field models for anisotropic interfaces. Physical Review E, 48(3):2016-2024, 1993.

[38] D. Min and H. Wong. The effect of strong surface energy anisotropy on migrating grainboundary grooves. Jour. Appl. Phys., 100:053523, 2006.

[39] L. Modica and S. Mortola. Il limite nella $\Gamma$-convergenza di una famiglia di funzionali ellittici. Boll Un Mat Ital, 14(5):526, 1977.

[40] W. W. Mullins. Theory of thermal grooving. J. Appl. Phys., 28:333-339, 1957.

[41] W. W. Mullins and R. F. Sekerka. Morphological stability of a particle growing by diffusion or heat flow. Jour. Appl. Phys., 34:323-329, 1963. 
[42] N. Nele Moelans, B. Blanpain, and P. Wollants. An introduction to phase-field modeling of microstructure evolution. Calphad, 32:268-294, 2008.

[43] B. S. Niethammer. Existence and uniqueness of radially symmetric stationary points within the gradient theory of phase transitions. European Journal of Applied Mathematics, 6(01), 1995.

[44] A. Novick-Cohen. Triple-junction motion for an Allen-Cahn/Cahn-Hilliard system. Physica D, 137:1-24, 2000.

[45] A. Novick-Cohen. The Cahn-Hilliard equation. Handbook of differential equations: evolutionary equations, 4:931, 2008.

[46] N.C. Owen, J. Rubinstein, and P. Sternberg. Minimizers and gradient flows for singularly perturbed bi-stable potentials with a dirichlet dondition. Proc. R. Soc. Lond. A, 429:505532, 1990.

[47] R. L. Pego. Front migration in the nonlinear Cahn-Hilliard equation. Proc. Roy. Soc. Lond., 422:261-278, 1989.

[48] S. Puri and K. Binder. Surface effects on spinodal decomposition in binary mixtures and the interplay with wetting phenomena. Physical Review E, 49:5359, 1994.

[49] A. Rätz, A. Ribalta, and A. Voigt. Surface evolution of elastically stressed films under deposition by a diffuse interface model. Journal of Computational Physics, 214(1):187-208, 2006.

[50] C. Redon, J. B. Brzoska, and F. Brochard-Wyart. Dewetting and slippage of microscopic polymer films. Macromolecules, 27(2):468-471, 1994.

[51] G. Reiter and A. Sharma. Auto-optimization of dewetting rates by rim instabilities in slipping polymer films. Phys. Rev. Lett., 87(16):166103, 2001.

[52] F. Ruffino and M. G. Grimaldi. Self-organized patterned arrays of au and ag nanoparticles by thickness-dependent dewetting of template-confined films. Journal of Materials Science, 49:5714-5729, 2014.

[53] D. N. Sibley, A. Nold, and S. Kalliadasis. Unifying binary fluid diffuse-interface motion laws in the sharp-interface limit. Jour. Fluid Mech., 736:5-43, 2013.

[54] J.E. Taylor and J.W. Cahn. Linking anisotropic sharp and diffuse surface motion laws via gradient flows. Jour. Stat. Phys., 77:183-197, 1994.

[55] J.E. Taylor and J.W. Cahn. Diffuse interfaces with sharp corners and facets: Phase field models with strongly anisotropic surfaces. Physica D, 112:381-411, 1998.

[56] C. V. Thompson. Solid-state dewetting of thin films. Annu. Rev. Mater. Res., 42:399-434, 2012.

[57] C. V. Thompson and J. Ye. Mechanisms of complex morphological evolution during solidstate dewetting of single-crystal nickel thin films. Appl. Phys. Let, 97:071904, 2010.

[58] C. V. Thompson and J. Ye. Anisotropic edge retraction and hole growth during solid-state dewetting of single crystal nickel thin films. Acta Mat., 59:582-589, 2011. 
[59] W. L. Winterbottom. Equilibrium shape of a small particle in contact with a foreign substrate. Acta Metall., 15:303-310, 1967.

[60] S. Wise, J. Kim, and J. Lowengrub. Solving the regularized, strongly anisotropic CahnHilliard equation by an adaptive nonlinear multigrid method. Jour. Comp. Phys., 226:414446, 2007.

[61] H. Wong, P. W. Vorhees, M. J. Miksis, and S. H. Davis. Periodic mass shedding of a retracting solid film step. Acta Mater., 48:1719-1728, 2000.

[62] T. Young. An essay on the cohesion of fluids. Philos. Trans. Roy. Soc. London, 95:65-87, 1805.

[63] P. Yue, J.J. Feng, C Liu, and J Shen. A diffuse-interface method for simulating two-phase flows of complex fluids. J. Fluid Mech., 515:293-317, 2004.

[64] P. Yue, C. Zhou, and J.J. Feng. Sharp-interface limit of the Cahn-Hilliard model for moving contact-lines. J. Fluid Mech., 645:279-294, 2010.

[65] R.V. Zucker, G. H. Kim, W. C. Carter, and C. V. Thompson. A model for solid-state dewetting of a fully-faceted thin film. C. R. Phys., 14:564-577, 2013. 